\title{
Rendezvous and Docking Strategy for Crewed Segment of the Asteroid Redirect Mission
}

\author{
Heather D. Hinkel ${ }^{1}$, Scott P. Cryan ${ }^{2}$, Christopher D'Souza PhD ${ }^{3}$, David P. Dannemiller ${ }^{4}$, Jack P. Brazzel ${ }^{5}$, Gerald L. \\ Condon ${ }^{6}$, William L. Othon ${ }^{7}$, Jacob Williams $^{8}$, \\ NASA-JSC, Houston, TX, 77058
}

\begin{abstract}
This paper will describe the overall rendezvous, proximity operations and docking (RPOD) strategy in support of the Asteroid Redirect Crewed Mission (ARCM), as part of the Asteroid Redirect Mission (ARM). The focus of the paper is on the crewed mission phase of ARM, starting with the establishment of Orion in the Distant Retrograde Orbit (DRO) and ending with docking to the Asteroid Redirect Vechicle (ARV). The paper will detail the sequence of maneuvers required to execute the rendezvous and proximity operations mission phases along with the on-board navigation strategies, including the final approach phase. The trajectories to be considered will include target vehicles in a DRO. The paper will also discuss the sensor requirements for rendezvous and docking and the various trade studies associated with the final sensor selection. Building on the sensor requirements and trade studies, the paper will include a candidate sensor concept of operations, which will drive the selection of the sensor suite; concurrently, it will be driven by higher level requirements on the system, such as crew timeline constraints and vehicle consummables. This paper will address how many of the seemingly competing requirements will have to be addressed to create a complete system and system design. The objective is to determine a sensor suite and trajectories that enable Orion to successfully rendezvous and dock with a target vehicle in trans lunar space. Finally, the paper will report on the status of a NASA action to look for synergy within RPOD, across the crewed and robotic asteroid missions.
\end{abstract}

\section{Introduction}

$\mathrm{T}$ HIS paper will describe the overall rendezvous, proximity operations and docking (RPOD) strategy in support o the Asteroid Redirect Crewed Mission (ACRM), as part of the Asteroid Redirect Mission (ARM). This paper will focus on the crewed mission phase of the ARM, starting with the establishment of Orion in the Distant Retrograde Orbit (DRO) and ending with docking to the Asteroid Redirect Vehicle (ARV). Details of the maneuver sequence to execute the rendezvous and proximity operations mission phases as well as ground and onboard navigation strategies are included. Sensor requirements for rendezvous and docking and various trade studies for sensor selection will be discussed. A candidate sensor concept of operations will build on the sensor requirements,

\footnotetext{
${ }^{1}$ Agency AR\&D Commonality Lead, EG6, 2101 NASA Parkway, Houston, TX 77058/EG6.

${ }^{2}$ Orion Relative Navigation Hardware Subsystem Manager, EG2, 2101 NASA Parkway, Houston, TX 77058 /EG2

${ }^{3}$ Orion Relative Navigation Software Subsytem Manager, EA, 2101 NASA Parkway, Houston, TX 77058/EG6, Senior Member AIAA.

${ }^{4}$ Aerospace Technologist, Mission Operations Directorate, 2101 NASA Parkway, Houston, TX 77058/ DM4, AIAA Senior Member

${ }^{5}$ Orion GN\&C Orbit Mode Team Lead and Rendezvous Proximity Operations and Docking Lead, EA, 2101 NASA Parkway, Houston, TX 77058/EG6, Senior Member AIAA

${ }^{6}$ Senior Aerospace Engineer, Aeroscience and Flight Mechanics Division, 2101 Nasa Pkwy, Houston, TX 77058/EG5, AIAA Member Grade.

${ }^{7}$ Deputy Chief GNC Development and Test Branch and iPAS Project Manager, EA, 2101 NASA Parkway, Houston, TX 77058 /EG2

${ }^{8}$ Deputy Chief GNC Development and Test Branch and iPAS Manager, ERC Inc., NASA Parkway, Houston, TX 77058
} 


\section{A. Overview of ARM}

NASA is developing two mission concepts for the Asteroid Redirect Mission (ARM) The first concept uses a robotic spacecraft to capture a whole small near-Earth asteroid and the second uses largely the same robotic spacecraft to capture a cohesive mass from a larger asteroid. In both mission concepts, the asteroid mass would be redirected into a stable orbit around the Moon. Astronauts aboard the Orion spacecraft launched on the Space Launch System (SLS) would rendezvous with the vehicle with the captured asteroid mass in lunar orbit and collect samples for return to Earth. NASA's activities in human exploration, space technology, and space science are being leveraged and integrated in order to make progress toward a range of objectives.

\section{B. Overview of how this $R \& D$ is different from previous missions}

NASA has flown manned rendezvous and docking (R\&D) missions since Gemini and many of the techniques pioneered by Gemini and used in Apollo and Shuttle are still applicable today. They are all based upon a rendezvous between two vehicles operating in a near-circular orbit in a strong central gravity field. However, neither of these factors are applicable to the ARM. Not only will the rendezvous be in a Distant Retrograde Orbit (DRO) (or other three-body near-equilibrium orbit), but the normal 'near-circular' orbit paradigm does not apply; neither does the "elliptical" orbit paradigm pioneered by Schauner".

In light of this, the normal "coelliptic" or "stable orbit rendezvous" of the Shuttle and Apollo era are no longer relevant. Thus, the constraints and the 'protections' afforded by operating in the neighborhood of a strong central body are no longer in effect.

The concept of operations will be a bit different from that in Low Earth Orbit (LEO). For instantce, the initial relative motion will be linear, hence, it will be fairly intuitive for the crew and those on the ground tasked with monitoring the rendezvous. Secondly, the timeline can be easily altered without regard for lighting considerations (since the lighting will not change appreciably during the rendezvous, expected to last no more than 12 hours). Finally, the lack of appreciable orbital dynamics will allow for stationkeeping at any relative position, with no appreciable cost in fuel.

However, all is not lost. In fact, the relative dynamics of two vehicles in a DRO are very nearly that of "fieldfree" space. Over short periods (i.e. hours), the two vehicles' relative motion can be approximated as follows:

Therefore, the rendezvous targeting is fairly straightforward, following a straight-line path, with little to no curvature involved. Therefore, the only factors to consider are (1) the time for the rendezvous and (2) the acceptable relative velocities, which would be a function of the range and (3) abort considerations. Each of these considerations is influenced by the fact that the two vehicles are operating in nearly field-free space.

\section{Orion Requirements}

\section{A. Mission Design}

The Asteroid Redirect Crewed Mission (ARCM) Design Reference Mission (DRM) centers on the translunar transit, rendezvous, and docking of an SLS-launched Orion carrying two crew members to an asteroid previously captured and redirected to a DRO by the Asteroid Redirect Vehicle (ARV). A DRO is a highly stable orbit that was selected for long-term stability, Orion-SLS access and launch availability. DROs are stable orbits where an object can be "parked" and remain in orbit for over a hundred years with minimal orbit maintenance maneuvers. This stability is attractive to ensure the asteroid remains in place and it minimizes the orbit maintenance propulsion required for the ARV. A 71,433km X-axis DRO is within the performance capabilities of the Block 1 SLS and Orion and was selected to maximize launch opportunities.

The mission was designed with a minimum propellant mission approach (also known as a minimum delta- $\mathrm{V}$ mission design). A minimum delta- $\mathrm{V}$ mission uses less of the available propellant, preserving some delta- $\mathrm{V}$ capability to accommodate trajectory failures and add flexibility to mission operations. The resulting mission duration is extended by several days, but the trade of additional mission days and the consumables needed to sustain the crew for that extra duration was worth the trajectory failure risk mitigation provided by the minimum delta- $\mathrm{V}$ mission design. The duration of an example minimum delta- $\mathrm{V}$ reference mission is approximately 26 days and includes two planned Extra-Vehicular Activities (EVAs) to explore a captured asteroid in DRO near the moon. A feasible mission timeline for an example epoch is shown in Table 1, and the major mission phases are described in

${ }^{9}$ Tschauner, J., 'The Elliptic Orbit Rendezvous', Aerospace Sciences Conference, AIAA Paper 66-537. 
detail throughout the rest of this document. The actual mission duration can vary between approximately 24 to 30 days or more depending on the specific epoch chosen. The companion summary trajectory for the example mission timeline is in Error! Reference source not found. The ARCM Mission Design Document (MDD) provides a detailed discussion of the ARCM reference trajectory (ref. X: .

Following launch, ascent and translunar insertion, the seven-day transit to the DRO rendezvous point includes a Lunar Gravity Assist (LGA) maneuver on Flight Day 6 and docking to the ARV on Flight Day 8. At the DRO, the two crew members will perform two four-hour EVAs to demonstrate deep-space EVA capabilities and retrieve asteroid samples for Earth return. The DRM supports six days total time at the DRO for joint operations, including rendezvous, five days of docked operations and protection for a contingency day. Following undocking on Flight Day 13, a return flight of 12 days will include another LGA maneuver on Flight Day 19, concluding with entry and recovery on Flight Day 26.

Table 1: Example ARCM Timeline

\begin{tabular}{|c|l|}
\hline Flight Day & \multicolumn{1}{|c|}{ Event } \\
\hline $\mathbf{1}$ & Launch, Ascent, TLI \\
\hline $\mathbf{2 - 5}$ & $\begin{array}{l}\text { Outbound Translunar Cruise. Depress to 10.2 psi, suit checkout/EVA } \\
\text { dry run, rendezvous/docking preparations }\end{array}$ \\
\hline $\mathbf{5}$ & Lunar Gravity Assist and Lunar close approach \\
\hline $\mathbf{5 - 7}$ & Lunar to DRO Cruise \\
\hline $\mathbf{8}$ & Rendezvous and Docking \\
\hline $\mathbf{9}$ & EVA 1 \\
\hline $\mathbf{1 0}$ & Suit refurbishment, EVA 2 prep \\
\hline $\mathbf{1 1}$ & EVA 2 \\
\hline $\mathbf{1 2}$ & Contingency margin, Housekeeping, Departure Prep \\
\hline $\mathbf{1 3}$ & Undock and Departure \\
\hline $\mathbf{1 9}$ & DRO to Lunar Cruise \\
\hline $\mathbf{2 0 - 2 6}$ & Lunar Gravity Assist maneuver \\
\hline $\mathbf{2 6}$ & Inbound Translunar Cruise, cabin stow, repress to $14.7 \mathrm{psi}$ \\
\hline
\end{tabular}




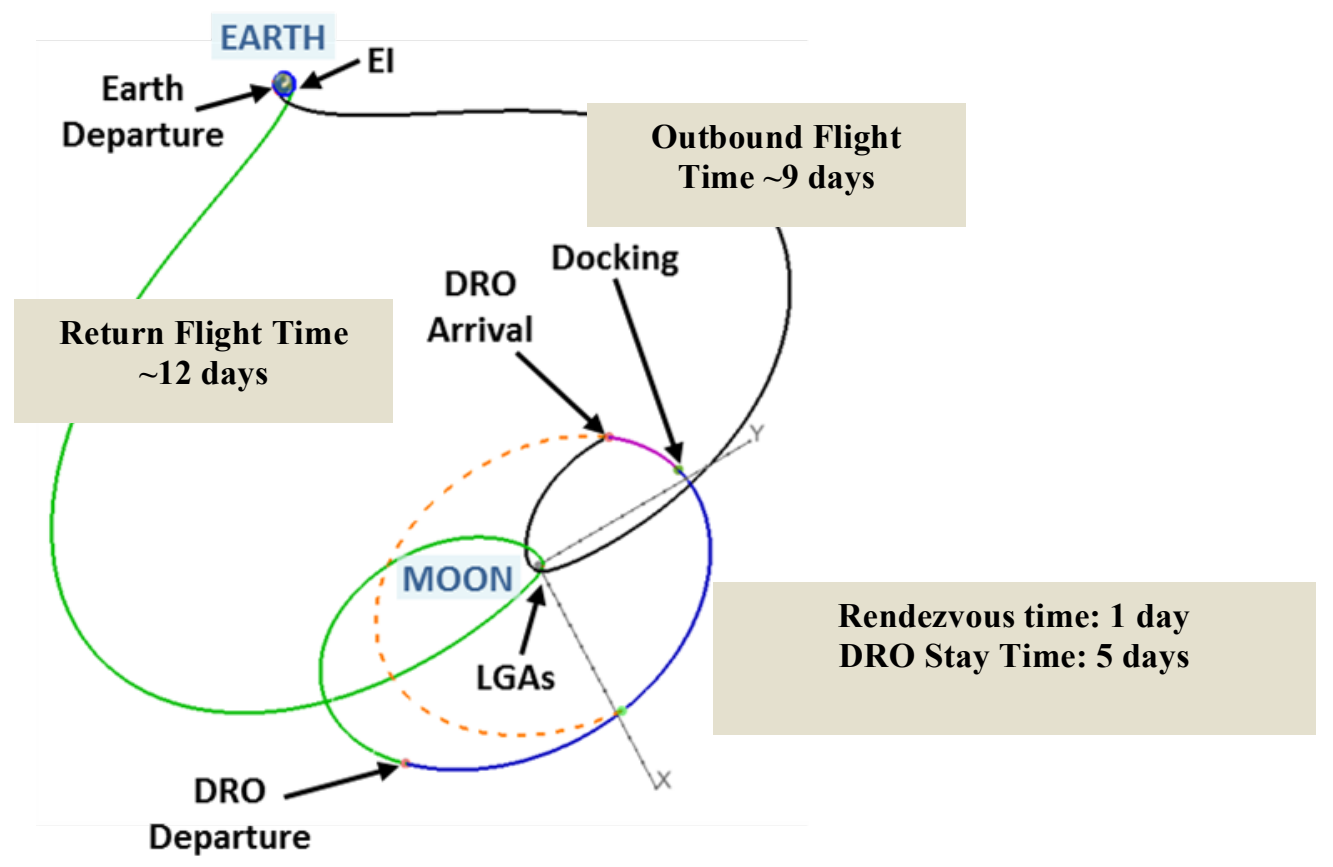

Nominal_Mission[0001].ideck

Figure 1: ARCM Trajectory Overview

\section{B. Sensor Requirements}

There were several factors that were the key driving requirements for the sensors. Of immediate importance were the needed measurements and their corresponding accuracy which were derived from both the mission and the relative navigation (RelNav) algorithm designs.

Orion's Guidance, Navigation and Control (GNC) levied requirements on the Communications and Tracking (C\&T) S-band equipment to provide a range and range-rate measurement to the target vehicle. The range and rangerate measurement requirements were based on legacy algorithms whereby accurate range and range-rate data, coupled with accurate bearing measurements would enable the RelNav filter to provide a reliably accurate state for performing the appropriate targeting burns during the rendezvous phase.

The physical aspects of the sensors were important. Power, mass and volume were driven by the available "footprint" on the vehicle, as well as the planned installation location on the vehicle. For Orion, the reuse of the RelNav hardware, the Vision Navigation Sensor (VNS), was critical. To enable reuse, it was determined the most appropriate location for the sensor was inside the Command/Crew Module (CM), in the docking tunnel, "looking" out the docking hatch window. This location offered the ability to keep the VNS in a "shirt-sleeve" environment and not be threatened or damaged by being physically exterior to the pressure vessel. This location also ensured that the sensors could be reused on future flights with minimal refurbishment. If the sensors had been mounted externally, there was a risk that they would be damaged during re-entry. External mounting also presented the vehicle designers with a challenging integration design to minimize the sensors' optics contamination during launch. Mechanized doors and glass portals were investigated but added mass and complex mechanisms that were deemed too costly.

Sensor measurement requirements were derived from the mission design and GNC designs. Initial GNC requirements dictated that Orion perform a targeting maneuver burn when the range to the target vehicle was roughly $2 \mathrm{~km}$, based on a LEO rendezvous, proximity operations and docking (RPOD) scenario with International Space Station (ISS). To ensure that a steady state, navigation solution was viable prior to the burn, the primary sensor, VNS, was required to commence measurements at a range of $5 \mathrm{~km}$ to the target, such that the sensor and filter would refine the measurement accuracy and navigation state respectively by the time Orion was $2 \mathrm{~km}$ from ISS. The sensor then had to provide measurements from acquisition/lock all the way until "docking" was achieved. 
In supporting the long-range through short-range RPOD phases, the sensors needed to have a reasonably sized field of view.

The cameras were initially specified to be $35^{\circ} \times 27^{\circ}$ to enable visual target vehicle detection at longer ranges. The final camera field of view will be dictated by Optical Navigation (OpNav) requirements, considering such factors and detector size, pixel pitch, focal length, etc. The last baseline for the docking camera dictated a field of view in the range of $35^{\circ} \times 26^{\circ}$ to $19^{\circ} \times 15^{\circ}$ over a minimum of 4 mega-pixel detector array. It is not unforeseen that public affairs office (PAO) will also have a say in what the camera requirements will be.

\section{Orion Concept of Operations}

\section{A. Trajectory Design}

A RPOD trajectory can be divided into the following 4 phases. 1) The far-field trajectory is driven primarily by gross motion from the Earth to the vicinity of the target. Relative motion between Orion and the ARV is not of primary concern, except that Orion moves toward the ARV. 2) The near-field rendezvous trajectory is designed to allow Orion relative sensors to observe the ARV and to begin to transition Orion to the docking axis. 3) Proximity operations brings the vehicles to very close proximity along the docking axis. 4) The docking phase involves 6-DOF control to bring the docking interfaces together.

\section{Far-field Trajectory}

The far-field rendezvous begins at launch and continues through DRO insertion or when relative navigation sensors acquire and track the target. The mid-field rendezvous covers the post DRO insertion through arrival at an offset position that begins the proximity operations (prox-ops) phase.

The DRO insertion target places the Orion on the DRO, either uprange or downrange of the ARV/asteroid target. The Orion performs all active mid-field and prox-ops maneuvers while the ARV remains quiescent. The mid-field maneuvers consist of at least two burns that follow a near rectilinear behavior and it concludes in an offset position from the ARV. It was assumed that the DRO insertion will be of similar magnitude to that of L2 Halo insertion. Analyses from Orion L2 Halo rendezvous indicated that the down-range error for Halo orbit insertion could be as large as $12 \mathrm{~km}$ for this feasibility study. Insertion at $30-50 \mathrm{~km}$ from ARV by Orion is feasible with the current absolute navigation accuracies. Navigation updates based on optical navigation and ground tracking will require at least two hours to obtain high-fidelity navigation solution to continue on with the rendezvous portion of RPOD. The ground tracking navigation would be the driver in terms of time required. Additional analyses have shown that with insertion at a range of $100 \mathrm{~km}$, the rendezvous phase can be completed in as little as $2.5 \mathrm{~h}$, assuming a closure rate of $10 \mathrm{~m} / \mathrm{s}$.

Use of S-band transponder based range and range-rate measurements can greatly decrease time to compute an accurate relative navigation state. Reliance on ground tracking derived state vectors coupled with optical based bearing measurements will increase time required to obtain accurate relative navigation states. Use of ground based state vector updates will likely require four to six hours to compute, thus impacting the RPOD timeline.

\section{Mid-Field Rendezvous Performance to Prox-ops Handoff}

The mid-field rendezvous in a DRO is much different than that of a rendezvous in LEO. An Earth orbit rendezvous can take as much as one to several days to complete, thus extending over many orbit revolutions. The DRO rendezvous, by contrast, occurs over a small fraction of the overall DRO period. Further, a DRO insertion downrange of $20-50 \mathrm{~km}$, from the Target, is a small distance relative to a 70,000 $\mathrm{km} \mathrm{x}$-axis distance for the orbit. Thus, a two-burn pair to close the $\sim 50-\mathrm{km}$ distance can be executed with near rectilinear targeting and flight (see Figure 1).

\section{Orion Mid-field Rendezvous}

Approximately 8 days and 10 hours after main engine cut-off (MECO), the Orion inserts into the DRO, solidifies its navigation state, and begins the process of closing the distance between it and the ARV. In order to close distance between two spacecraft, the chaser performs a simple two-burn sequence (see Figure 1). The Orion first performs a maneuver to close the distance gap and, after a selected time period, performs a second maneuver, at a selected offset distance, to match the velocity of the target vehicle (ARV). The Orion is then poised for final approach and docking maneuvers. Note that for this example, the distance offset at the final time is shown to be $10 \mathrm{~m}$. This distance was only used for demonstration purposes and could be modified to a larger value (100 m, 1,000 m, etc.) as dictated by operational requirements. 


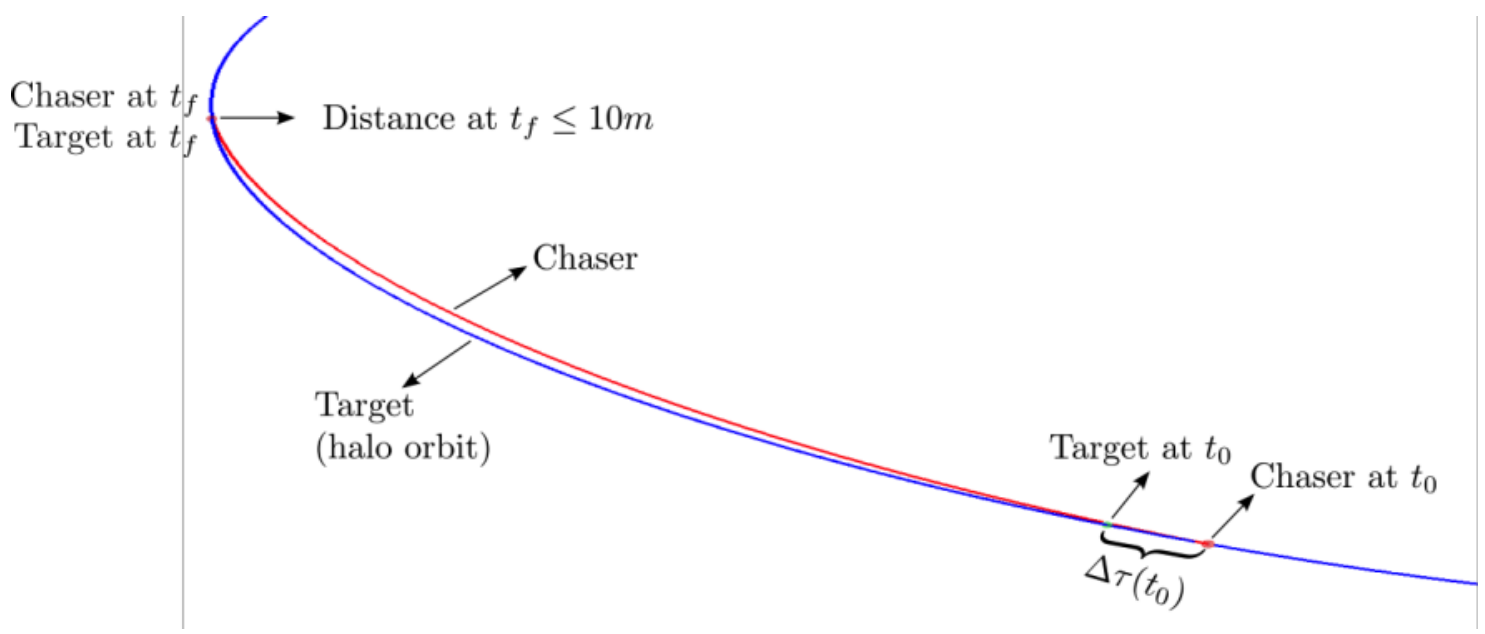

Figure 1. Two-maneuver rendezvous sequence.

The cost of this maneuver sequence is time-dependent. The more time allotted, the lower the $\Delta \mathrm{v}$ requirement. Figure 2 shows a performance trade for DRO orbits with $\mathrm{x}$-axis distances (from the moon) of $70,000 \mathrm{~km}$. The $\Delta \mathrm{v}$ cost is essentially the same whether the chaser is ahead of or behind the target at the initialization of the two-burn sequence.

Currently, the anticipated navigation error for insertion onto a DRO is $12 \mathrm{~km}$ in downrange and $1 \mathrm{~km}$ in crossrange $^{10}$, with a turn-around time for a navigation state update anticipated to be $<2 \mathrm{~h}$ assuming S-Band based range and range-rate and optically based bearing measurements are supplied to the relative navigation filter. Given these position errors, a $30-50 \mathrm{~km}$ downrange insertion target could be accommodated.

Figure 2 shows the downrange and uprange distance of a chaser from a target at DRO insertion along with corresponding angular distance. Three families of $\Delta \mathrm{v}$ costs represent a 6,12 , and 24 hour rendezvous (i.e. two-burn sequence).

Given 6 hours to complete the sequence, $5 \mathrm{~m} / \mathrm{s} \Delta \mathrm{v}$ can cover a closure distance of approximately $50 \mathrm{~km}$ (the higher end of the downrange distance between the ARV and Orion). Longer rendezvous times would result in a smaller $\Delta \mathrm{v}$ for the same distance. Thus, a $5 \mathrm{~m} / \mathrm{s}$ rendezvous budget was selected as a reasonable $\Delta \mathrm{v}$ for mid-field DRO rendezvous. The conclusion of this rendezvous phase leaves the chaser (Orion) at a prescribed offset state ${ }^{11}$ to begin the prox-ops to docking phase.

\footnotetext{
${ }^{10}$ Based on a conversation with JSC navigation expert, Chris D'Souza.

${ }^{11}$ Note that the target mid-field state allows the MPCV to pass the ARV without impact should there be no braking maneuver (in the rendezvous burn pair) executed during the planned prox-ops phase.
} 


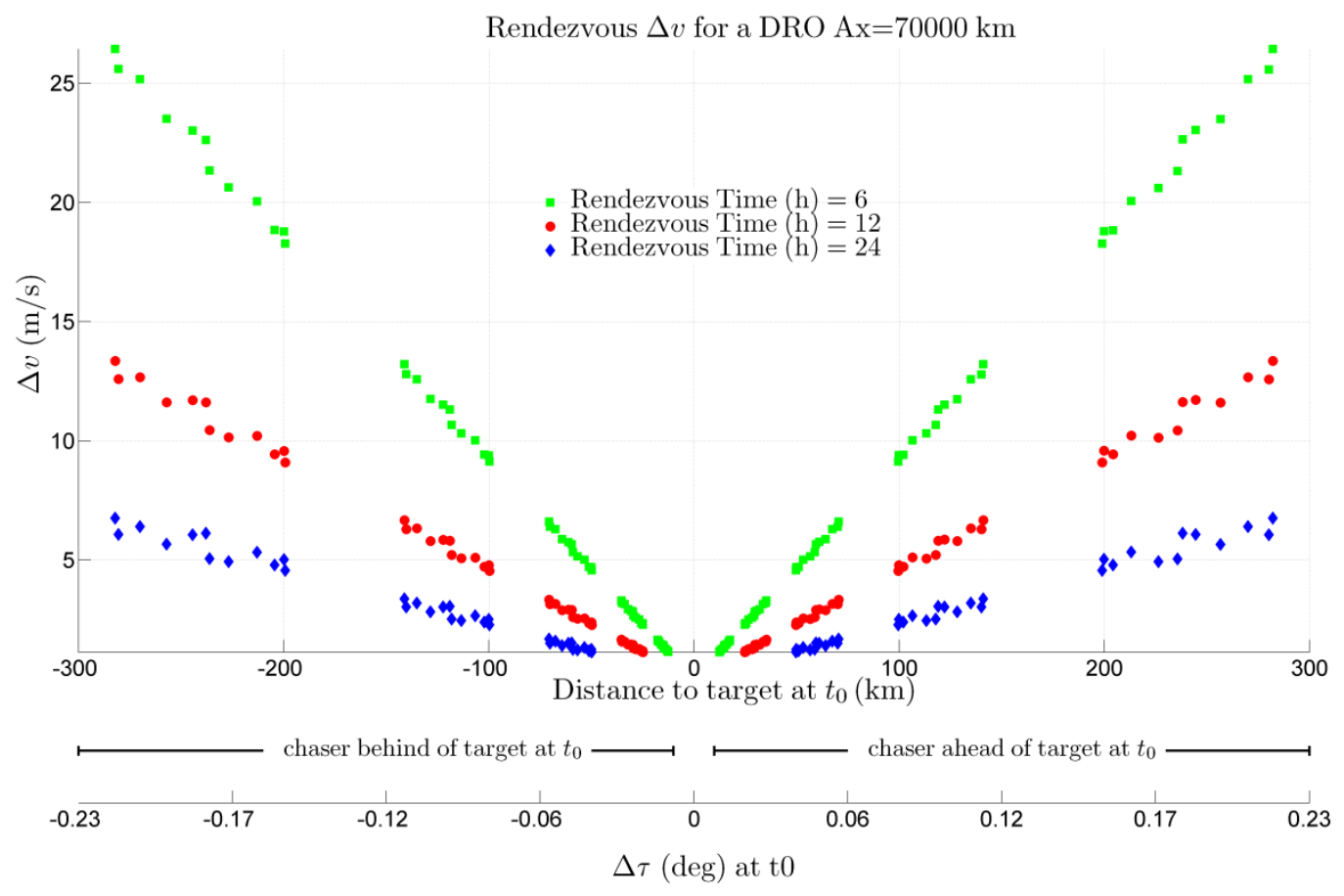

Figure 2. $\Delta v$ cost vs. initial target distance (or angle).

\section{Strategies for Transition from Far Field to Prox-ops Rendezvous}

This section contains a candidate approach for mid-field rendezvous maneuvers to a final prox-ops standoff distance from an ARV in a DRO. It also examines the consistency of a given approach for a rendezvous occurring at various positions on a DRO. It assumes a $20 \mathrm{~km}$ distance between Chaser (Orion) and Target (ARV) following DRO insertion and a $300 \mathrm{~m}$ offset distance from the Target as a transition to prox-ops. However, this $300 \mathrm{~m}$ distance is used as an example and can be modified to accommodate other rendezvous mission considerations in the future.

The $20 \mathrm{~km}$ to $300 \mathrm{~m}$ transfer consists of a minimum of 2 burns (one to begin closure between Chaser and the Target and one to zero out the relative velocity at the target offset position arrival). Figure 3 shows the $\Delta v$ cost of an approximately $2 \mathrm{~m} / \mathrm{s}$, two burn sequence as a function of initial range between the Chaser and Target offset position and the final $300 \mathrm{~m}$ offset distance. It is anticipated that additional maneuvers would be added to this two-burn sequence to provide for a more controlled, slower approach that contains natural collision avoidance given failed downrange maneuvers. 


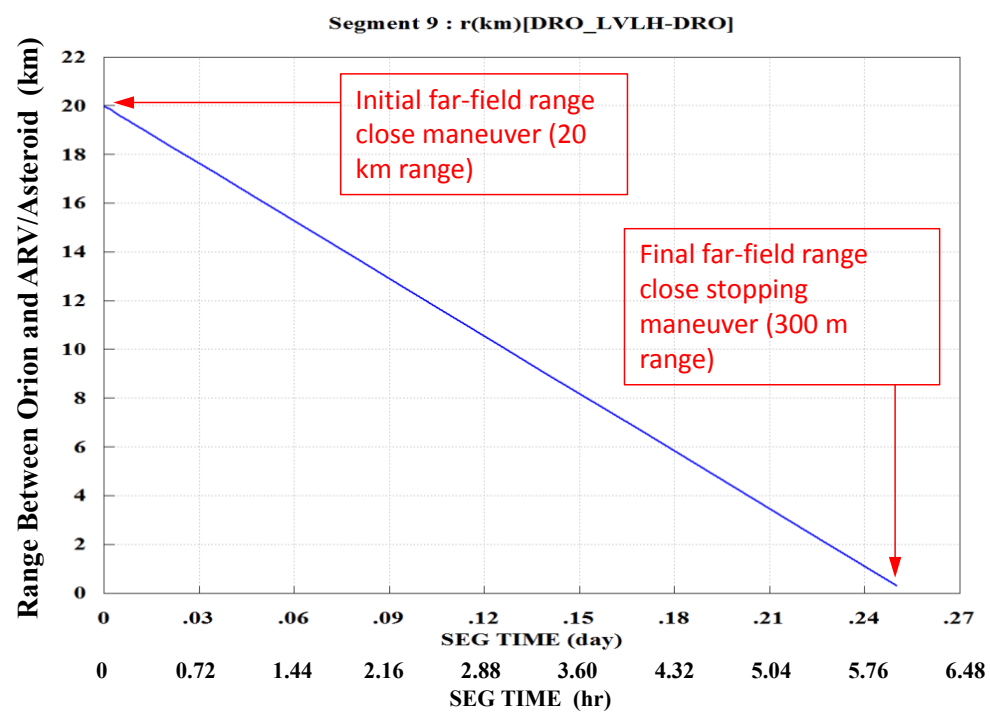

Figure 3. Orion to ARV/asteroid range distance during the two burn mid-field rendezvous flight phase. Total Dotal approximately $2 \mathrm{~m} / \mathrm{s}$.

A DRO Local Vertical, Local Horizontal (LVLH) reference frame was established for rendezvous and prox-ops design (see Figure 4). The DRO is shown in an Earth-Moon Rotating Frame. The LVLH frame(s) are shown in Inertial Moon-Centered Frame(s).

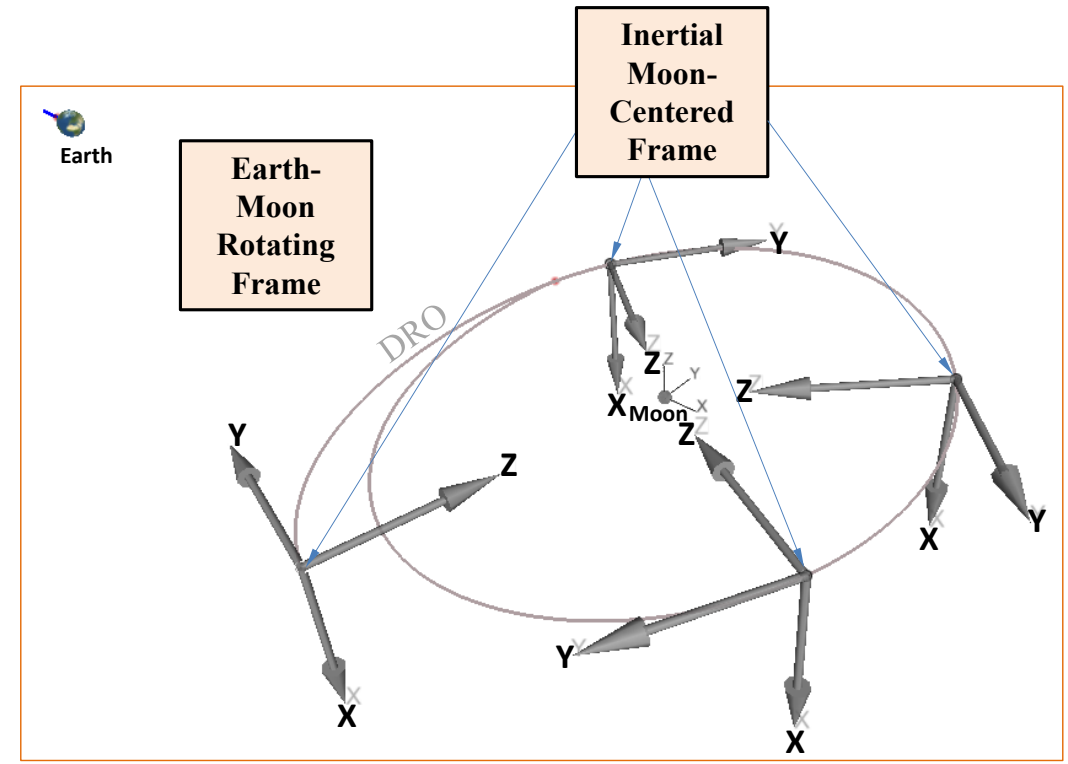

Figure 4. DRO LVLH Frame.

This frame provides relative motion between a target and chaser in a DRO (see Figure 5). The Moon Centered Inertial Frame consists of the following component directions:

$\mathrm{X}=$ Angular momentum direction (out of plane)

$\mathrm{Z}=$ Radial direction (to Moon)

$\mathrm{Y}=\operatorname{cross}(\mathrm{Z}, \mathrm{X})$

The Y-Z plane is the Earth-Moon (DRO) Plane.

American Institute of Aeronautics and Astronautics 


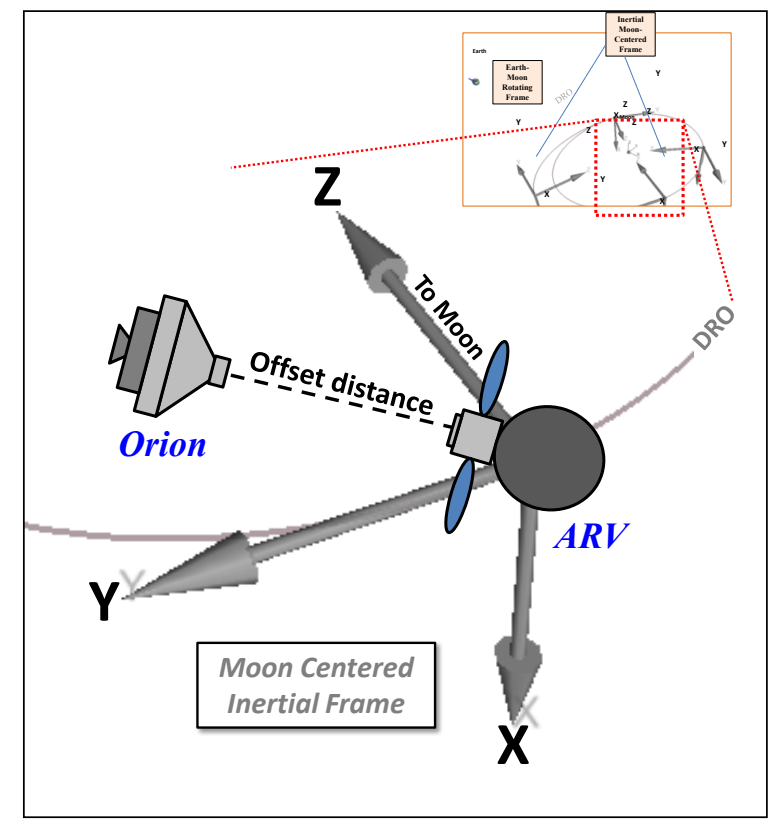

Figure 5. DRO LVLH frame with Orion offset from target

\section{Near-field Trajectory}

As described in section <page 2, Overview of how this R\&D is different from previous missions $>$, the DRO near-field environment (within order $100 \mathrm{~km}$ relative range) is different than low Earth orbit and low lunar orbit where RPOD has historically been performed. Those differences are factored into the near-field trajectory design that follows.

The ARV is the target vehicle. While docked, the ARV orients the stack (ARV/Orion) such that the tail of Orion is pointed to the sun, which is preferred by Orion for thermal control and power generation. This paper assumes the ARV is in the nominal docked attitude during rendezvous. This attitude seems to work well, but should not be considered a constraint. Further investigation may reveal advantages to another ARV attitude strategy.

As designed, the DRO is in the Earth-Moon plane. The angle between the Earth-Moon plane and the ecliptic (Sun-Earth plane) is about 5 degrees. Because the Earth, Moon, Sun, and ARV are all traveling in approximately the same plane, there will be periods when these objects might be in the field of view (FOV) of one of the optical sensors on Orion. Further analysis is needed to fully address control of bright objects for optical sensors passes.

The far-field trajectory dictates initial conditions for the near-field rendezvous trajectory. As shown in Figure12, the near-field trajectory starts with Orion approaching the ARV from the direction of the Moon. Figure 12 also shows the general LOS to the Earth and Moon. When Orion optical sensors are initially directed at the ARV, it is clear that the Earth and Moon will not be in the sensor FOV. This statement is only true for the initial pass. 


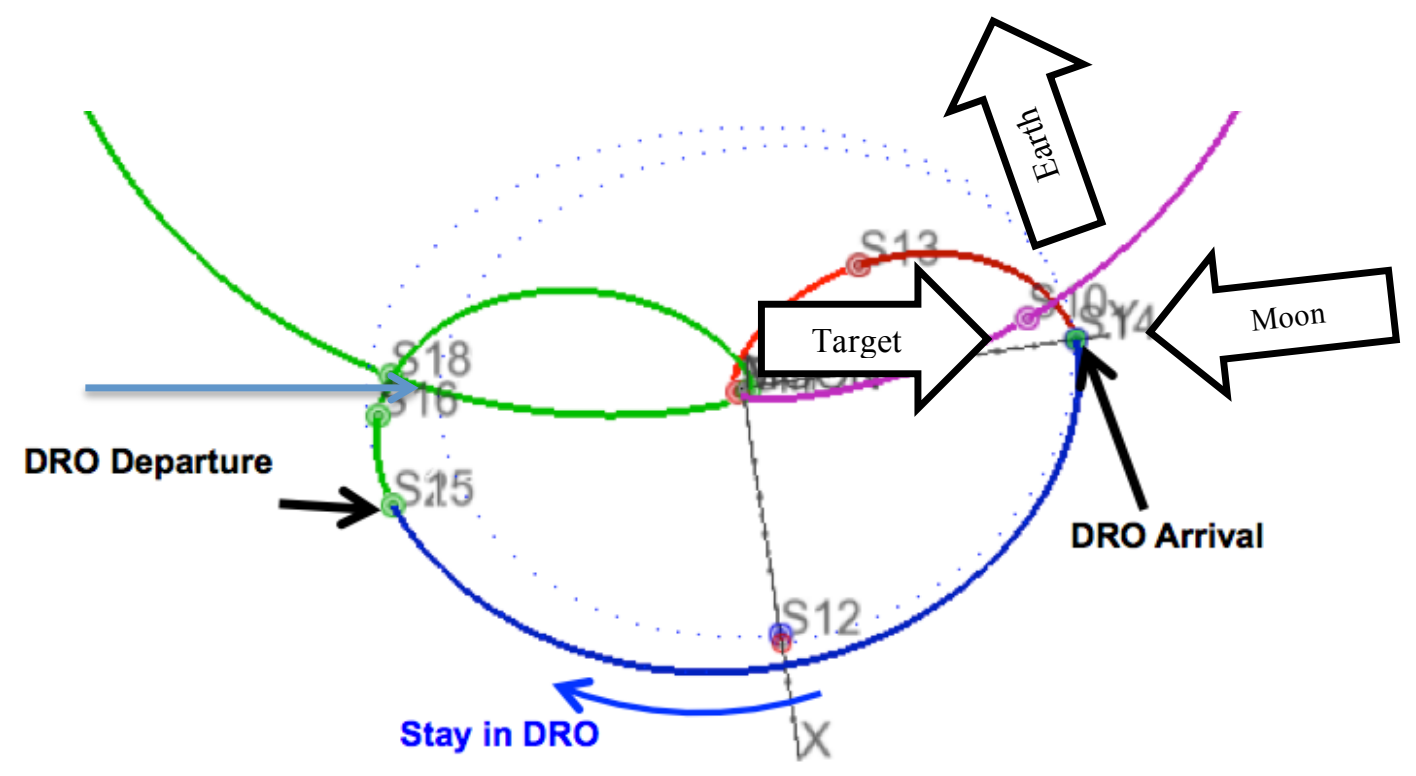

Figure 32. Title Goes Here

The near-field trajectory is shaped to deliver Orion to the docking axis. Because of the ARV attitude discussed earlier, the docking axis is oriented toward the sun. Figure 13 shows that the Orion performs a series of maneuvers to transition from the initial position (dictated by the far-field trajectory) to the docking axis. The offset shown in Figure 13 is sized by dispersions to allow passive abort. In other words, Orion can safely discontinue the rendezvous without having to perform abort maneuvers.

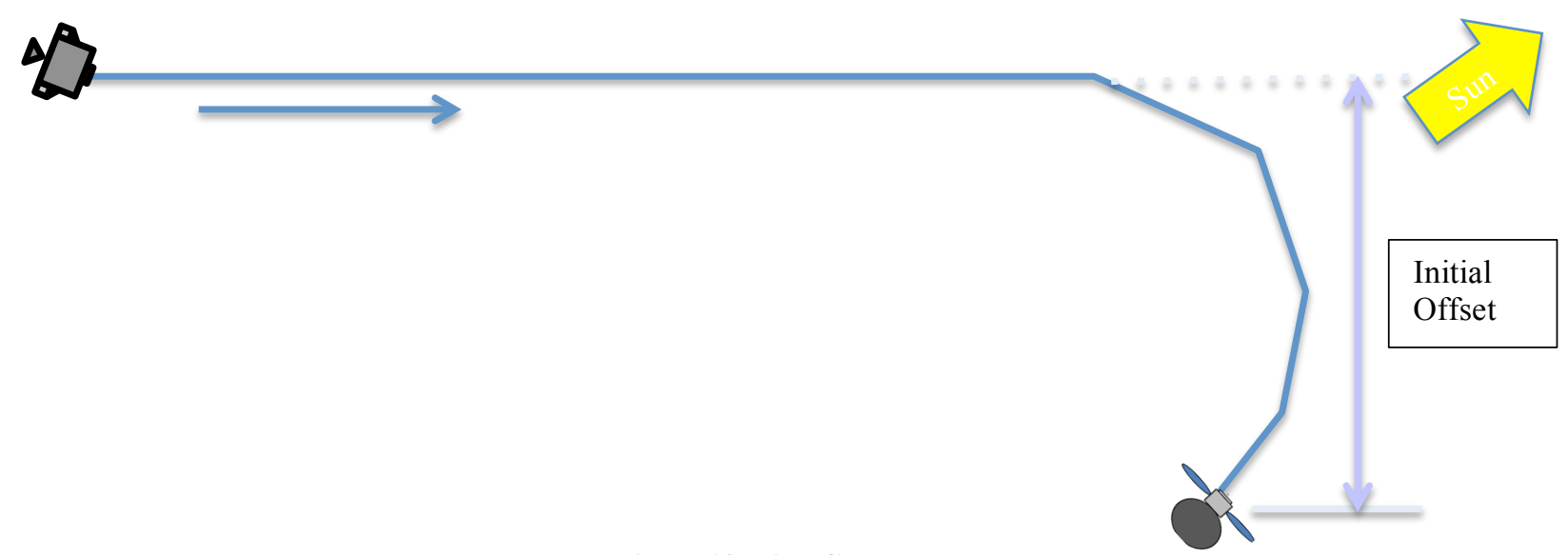

Figure 13. Title Goes Here

Figure 13 also shows an example direction to the Sun. Placing the initial offset as shown ensures that the LOS from Orion-to-ARV trends away from the Sun. Figure 14 shows a different direction to the Sun, and the resulting change of initial offset. Using this strategy of placing the initial offset (up-Sun), it is apparent that the maximum turn angle of the relative trajectory is 180 degrees. Later in this section, an example trajectory and maneuver sequence are provided for a 90 degree turn angle. 


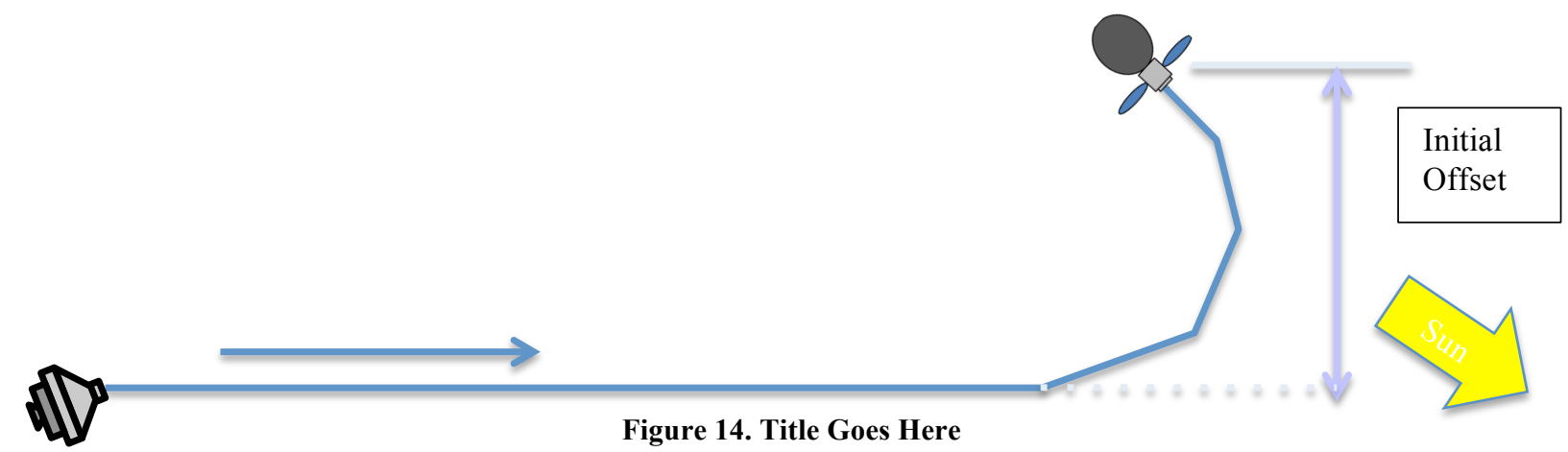

The maneuvers are constrained to a plane know as the Approach Plane (AP). A plane can be defined by a line and a point. The approach plane contains the docking axis (a line). This paper assumes that the approach plan also contains the initial position at the beginning of near-field rendezvous. The seems to work well but should not be considered a constraint. Further investigation may reveal advantages to rotating the AP about the docking axis, especially to avoid bright objects such as the Earth and Moon.

As described in section <Scott's sensor section>, the ARV cooperates with the Orion navigation sensors by providing a transponder for RF ranging, retro-reflectors for the light detection and ranging (LIDAR), and a docking target. The near-field trajectory is designed to satisfy the constraints of these sensors. For example, return signal strength from the retro-reflectors tends to decrease as the LIDAR gets farther off the boresight of the retro-reflector. This places a constraint of the trajectory at LIDAR acquisition.

As Orion approaches the ARV in the far-field, relative velocities are on the order of $200 \mathrm{~m} / \mathrm{s}$. At this velocity, Orion would be within relative sensor range for only a few minutes. The relative motion must be slowed down to allow more time.

Figure 15 shows the relative motion after the first rendezvous burn - Transition to Approach Plane (TAP). The TAP burn slows the relative velocity to less than $10 \mathrm{~m} / \mathrm{s}$, so is a large burn. It is performed using the OMS engine. The initial offset is maintained to preserve passive abort capability.

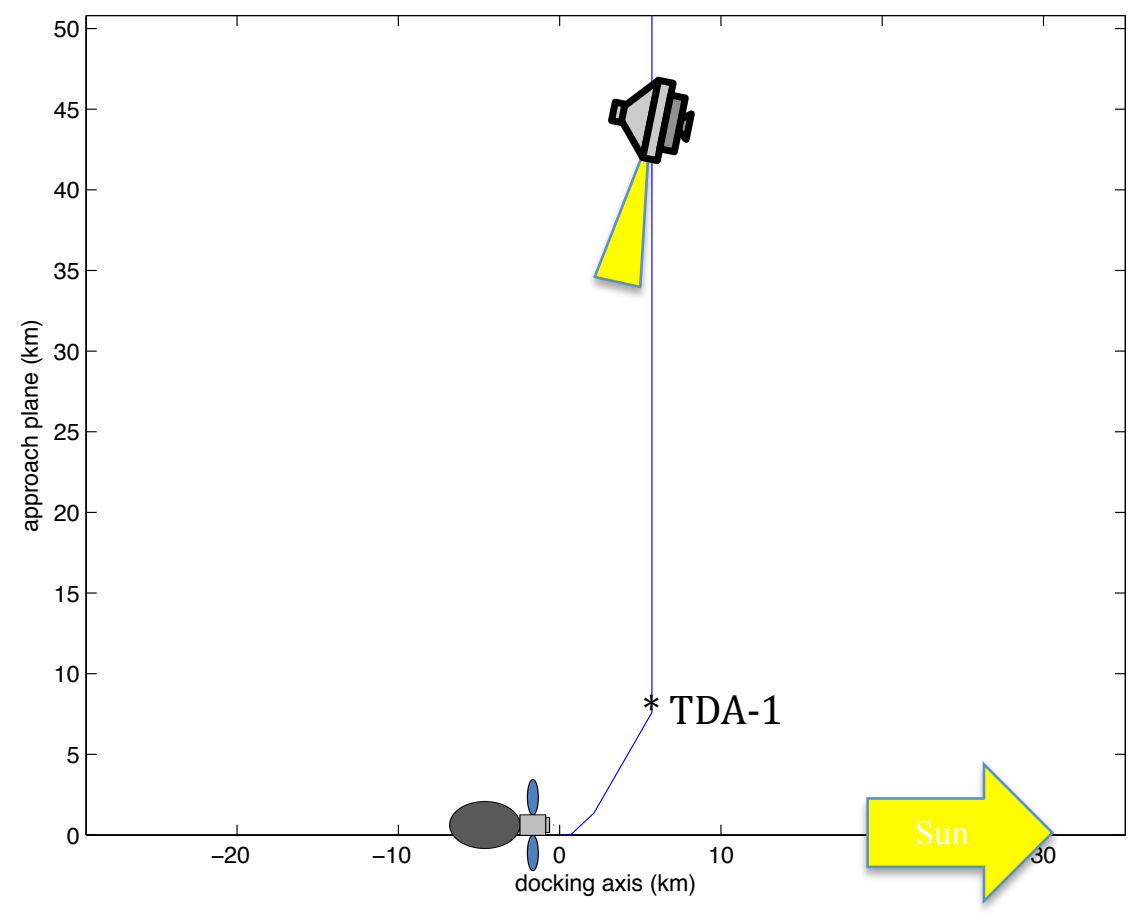

Figure 15. Title Goes Here

After the TAP burn, Orion is oriented to place the ARV in the FOV of one of the star trackers. Roll about the star tracker line of sight (LOS) is used to optimize Orion power generation, thermal control, and communication. Star 
tracker bearing and RF ranging measurements are processed by the RelNav filter to improved the estimate of relative state. The post-TAP trajectory leg shown in Figure 15 provides 90 minutes for relative measurements. Future dispersion analysis will be used to place mid-course maneuver(s) along this leg.

Figure 16 shows the remaining rendezvous maneuvers - Transition to Docking Axis (TDA) 1, 2 and 3. TDA-1 is performed using the Orion $+X$ (or Aux) engines, after which Orion is oriented to point $+X$ toward the ARV to allow bearing measurements from the docking camera to be used with Rf ranging measurements in the RelNav filter. Roll about the $+\mathrm{X}$ LOS is used to optimize Orion power, thermal, and communication.

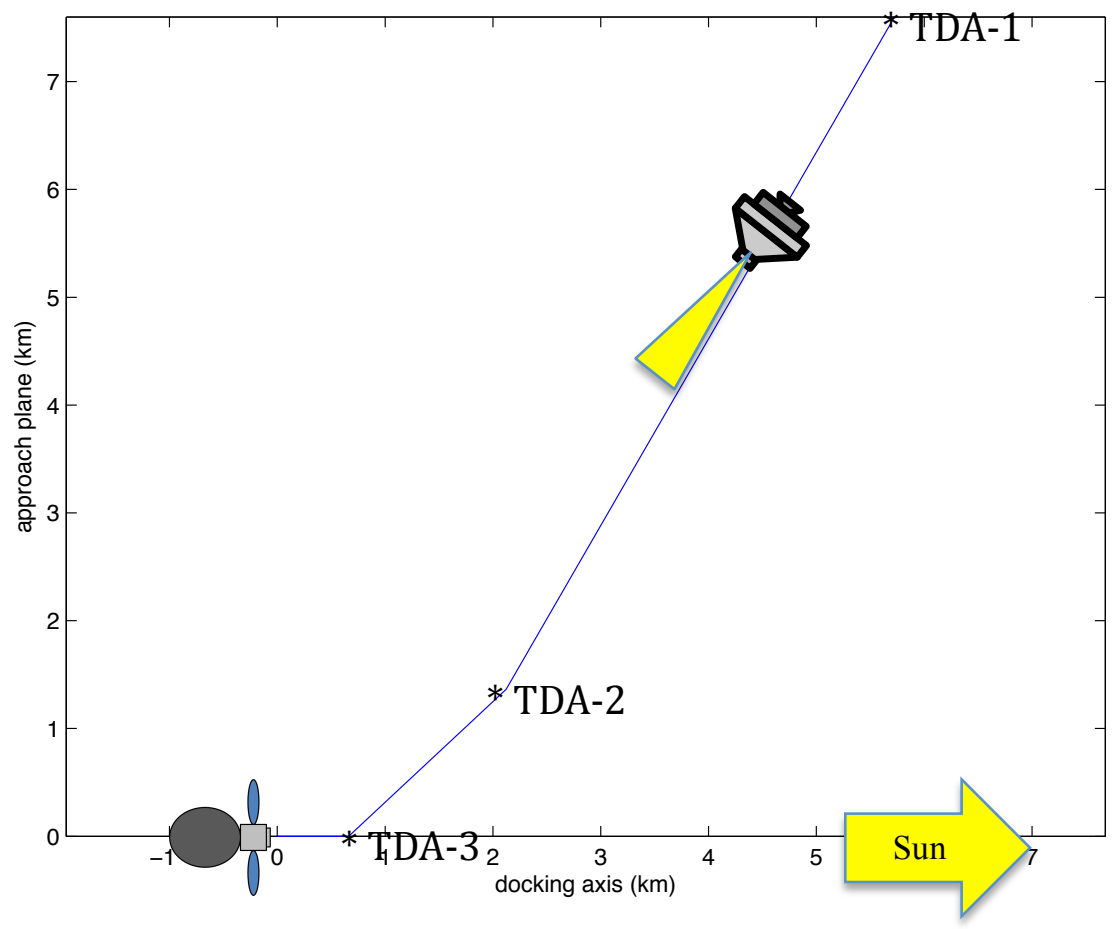

Figure 16. Title Goes Here

Figure 17 shows TDA-2 and 3 which are performed using the Orion Reaction Control System (RCS) engines in a multi-axis burn mode. This allows the docking camera and LIDAR to remain pointed toward the ARV. Seventeen minutes after TDA-2, LIDAR acquisition range of $2 \mathrm{~km}$ is reached. LIDAR range and bearing measurements are processed by the RelNav filter. The time between LIDAR acquisition and TDA-3 is 50 minutes. Future dispersion analysis will be used to place mid-course maneuver(s) along this leg. 


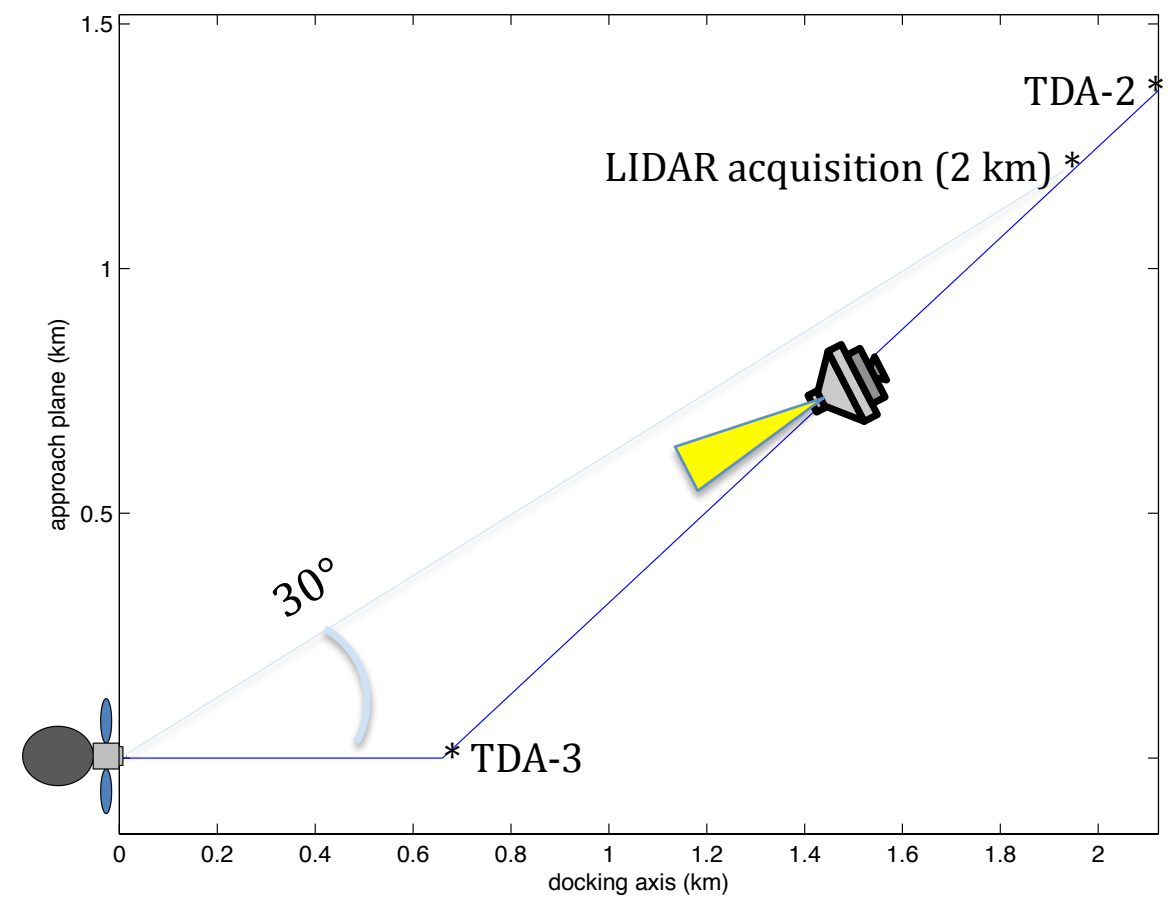

Figure 17. Title Goes Here

So far, roll about the sensor LOS (either star tracker of $+\mathrm{X}$ ) has been used to optimize Orion power, thermal, and communication. As Orion nears TDA-3 and the docking axis, Orion naturally tends to the preferred tail-to-Sun attitude. Roll about the $+\mathrm{X}$ is now adjusted to align the clocking angle of the two docking mechanisms on Orion and ARV.

TDA-3 places Orion on the docking axis at approximately $660 \mathrm{~m}$, and is the end of near-field rendezvous.

Dispersion analysis has not yet been performed but will eventually be used to place mid-course maneuver(s) to control the relative trajectory.

Table A. Title Goes Here

\begin{tabular}{|l|l|l|l|}
\hline $\begin{array}{l}\text { Range } \\
(\mathbf{m})\end{array}$ & $\begin{array}{l}\text { Brake to } \\
(\mathbf{m} / \mathbf{s})\end{array}$ & $\begin{array}{l}\text { Time to Capture } \\
(\mathbf{m i n})\end{array}$ & Note \\
\hline 660 & -.3 & 58.9 & \\
\hline 300 & -.2 & 38.9 & \\
\hline 100 & -.1 & 22.2 & \\
\hline 10 & 0 & $7.2(5$ min hold) & docking mechanism $\Delta$ temp, check Pose, GO \\
\hline 10 & -.075 & 2.2 & \\
\hline 0 & N/A & 0 & Capture \\
\hline
\end{tabular}

6. Proximity Operations and Docking 
Table A shows the Proximity Operations timeline and Braking gates. Orion arrives on the docking axis at approximately $660 \mathrm{~m}$ with a relative closure rate of $-0.3 \mathrm{~m} / \mathrm{s}$. At this point, the time to capture is about an hour. The LIDAR continues to track the target vehicle and provides relative navigation updates. Orion docking lights will illuminate the docking target so that the crew can visually monitor and maintain situational awareness. As Orion continues along the docking axis, at $300 \mathrm{~m}$, the closure rate is reduced to $-0.2 \mathrm{~m} / \mathrm{s}$. And similarly, at $100 \mathrm{~m}$ the closure rate is reduced to $-0.1 \mathrm{~m} / \mathrm{s}$. At $10 \mathrm{~m}$, Orion performs a hold maneuver for approximately five minutes to perform final checkouts prior to docking. Checkouts include docking mechanism preparation as well as verification of relative navigation to 6-DOF pose mode. Once all checkouts are complete and verified, Orion increases the closure rate to $-0.075 \mathrm{~m} / \mathrm{s}$ continues to dock. At this point, Orion is approximately two minutes from docking contact. Orion maintains the $-0.75 \mathrm{~m} / \mathrm{s}$ closure rate up until contact.

During proximity operations and docking, plume impingement and contamination from the Orion thrusters on the ARV solar arrays has to be addressed. Plume impingement and contamination analysis has not yet been performed. While the analysis might result in some limitation of Orion RCS jet firings at close range, the limitations are not expected to be significant.

Figure $<>$ shows a summary of the maneuvers, attitude profile, and sensor usage during the day of rendezvous.

\section{B. DRO Sub-Studies}

\section{Relative Motion on a DRO}

The purpose of this sub-study was to gain insight as to the natural drift of a spacecraft relative to a target, for a given initial offset position from the target (i.e., along the plus and minus LVLH axes). This information will aid in selecting a rendezvous approach, which will be influenced by crew safety. In this case, the concern would be the possibility of the chaser (Orion) impacting the target after a successful insertion onto a prox-ops offset position followed by an uncontrolled free drift (e.g., possibly due to engine failure after this insertion).

Figure 6 shows an Orion spacecraft placed at a fixed $300 \mathrm{~m}$ distance from the center of the LVLH frame (the target, ARV/asteroid location). The Orion spacecraft initially carries a zero velocity relative to the target. This is followed by a free drift lasting 4 days. The propagation results show that, for this example, positions on the $\mathrm{X}$ (angular momentum, out of plane) direction drift toward a possible collision with the ARV. However, propagation in the $\mathrm{Z}$ (radial) and Y (velocity) directions provide movement away from the ARV resulting in little or no threat of recontact.

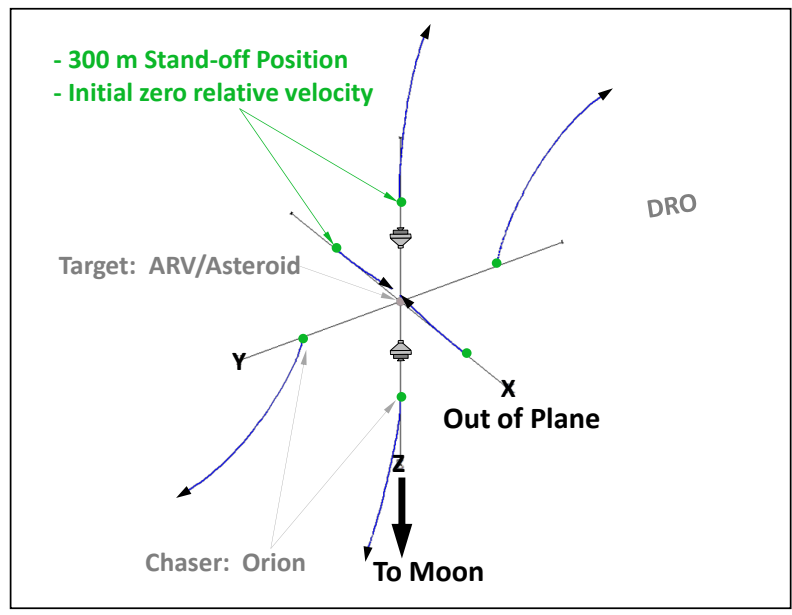

Figure 6. Orion drift relative to rendezvous target, for a 4-day propagation.

This drift behavior appears to be consistent, for the given $300 \mathrm{~m}$ relative target offset example, with varying insertion epochs or DRO phase angles (see Figure 7). Again here, the out of plane offset positions pose the largest possibility of recontact with no maintenance maneuvers. The radial offset positions are preferred due to the natural collision avoidance between the Chaser and Target for a 4 day propagation. 


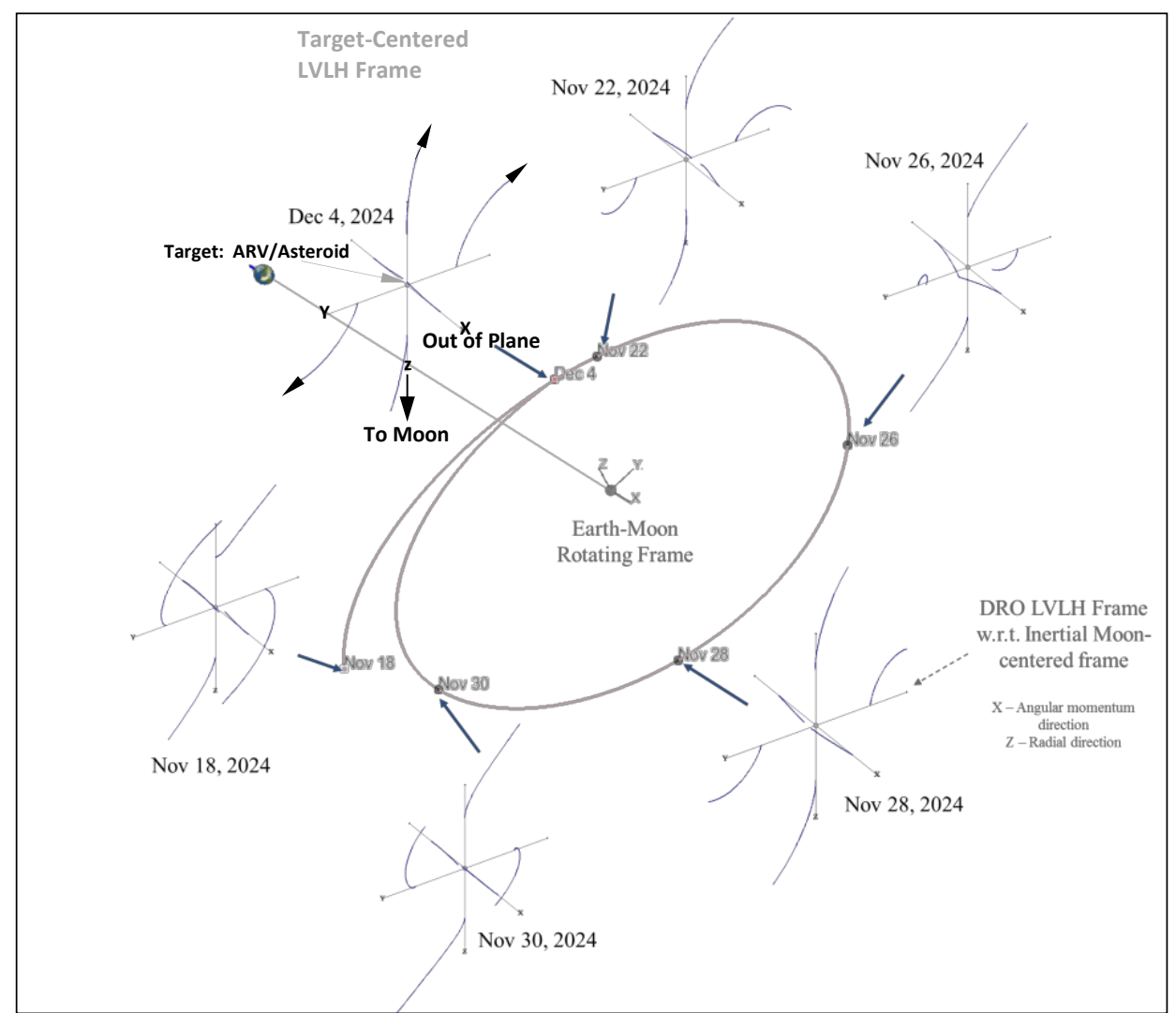

Figure 7. Orion drift relative to rendezvous target for a 4-day propagation with varying DRO insertion epochs or DRO phase angles.

A composite view of drift, from an initial $300 \mathrm{~m}$ offset position with a subsequent 10-day propagation time (see Figure 8) reinforces that the out-of-plane direction (x-axis) is not suitable for a DRO insertion, since it will naturally tend to approach or collide with the ARV if no subsequent maneuvers are performed. Some close approaches are also possible with some phase angles with a y-axis offset point. The $\mathrm{Z}$ (radial) direction (above or below the ARV) as the DRO insertion point, provides the best natural anti-collision effect as the spacecraft naturally drifts away from the DRO.

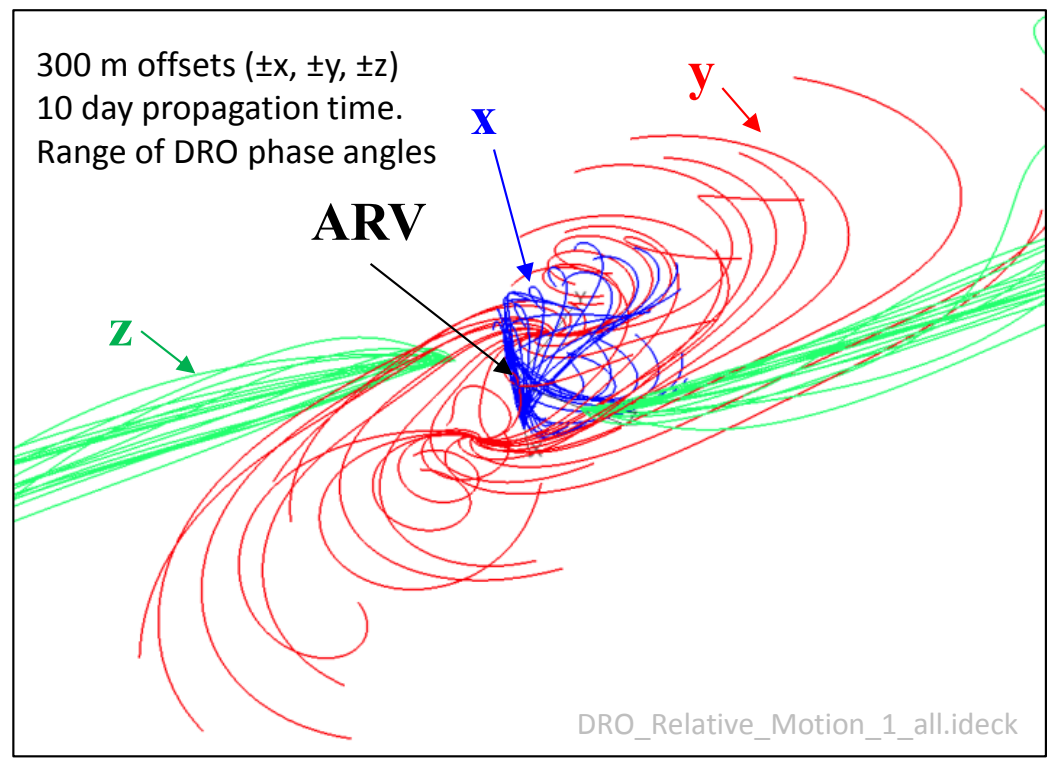

15

American Institute of Aeronautics and Astronautics 
Figure 8. 10-day propagation from $300 \mathrm{~m}$ offset positions on the $\mathrm{X}, \mathrm{Y}$, and $\mathrm{Z}$ axes.

\section{While On A DRO, Transfer From $20 \mathrm{~km}$ To a $300 \mathrm{~m}$ Offset Postion}

This sub-study is designed to assess the $\Delta \mathrm{v}$ cost of a mid-field transfer (e.g., from $20 \mathrm{~km}$ DRO insertion range [from ARV/asteroid] to $300 \mathrm{~m}$ prox-ops offset range). It also attempts to assess the flight path prior to and after the stopping maneuver (i.e., at $300 \mathrm{~m}$ prox-ops range to Target).

It is assumed for this study that: the DRO insertion offset at $t_{0}$ (magnitude and direction) is $20 \mathrm{~km}(+\mathrm{Z})$ [radial below DRO orbit]; the wait time before the first burn (Rendezvous 1 maneuver) is performed is $1 \mathrm{hr}$ (accounts for navigation state update and maneuver preparation); the transfer time $\Delta t$ from $20 \mathrm{~km}$ to $300 \mathrm{~m}$ is approximately 6 hrs; the final offset distance at $t_{2}$ (magnitude and direction) is $300 \mathrm{~m}$ [direction depends on ARV orientation and desired Orion insertion location].

These transfer results are shown in Figures $9 \mathrm{a}-9 \mathrm{e}$. The $\Delta \mathrm{v}$ cost for all cases is about $2 \mathrm{~m} / \mathrm{s}$. The 2-burn maneuver sequence has potential collision issues if the final offset point is too near the radial (Z) axis. The 2 burn sequence targets away from the radial $(Z)$ direction.

Rectilinear trajectories are very similar and relatively independent of direction of position on $300 \mathrm{~m}$ "circle" relative to the ARV/asteroid target as well as (angular phase) location on the DRO.

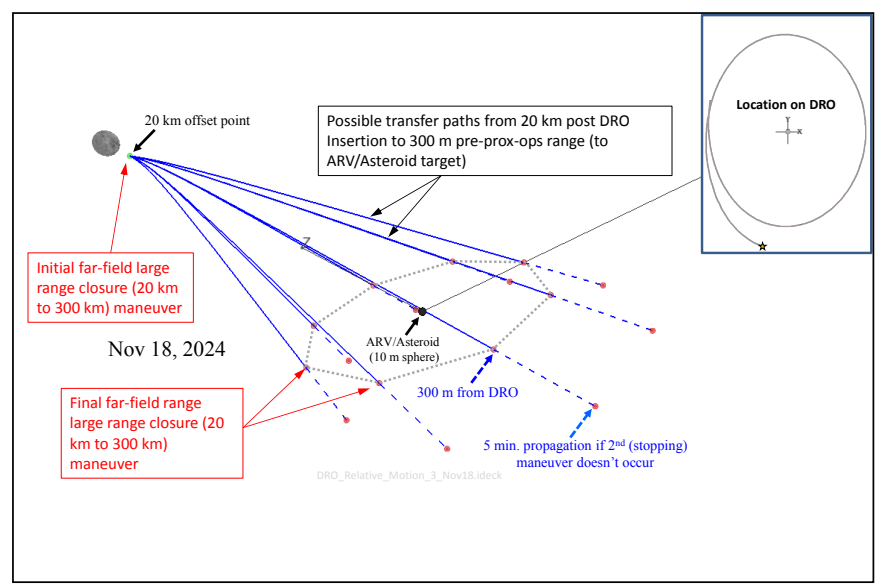

Figure 9a. November 18, 2024 maneuver from $20 \mathrm{~km}$ to $300 \mathrm{~m}$ offset

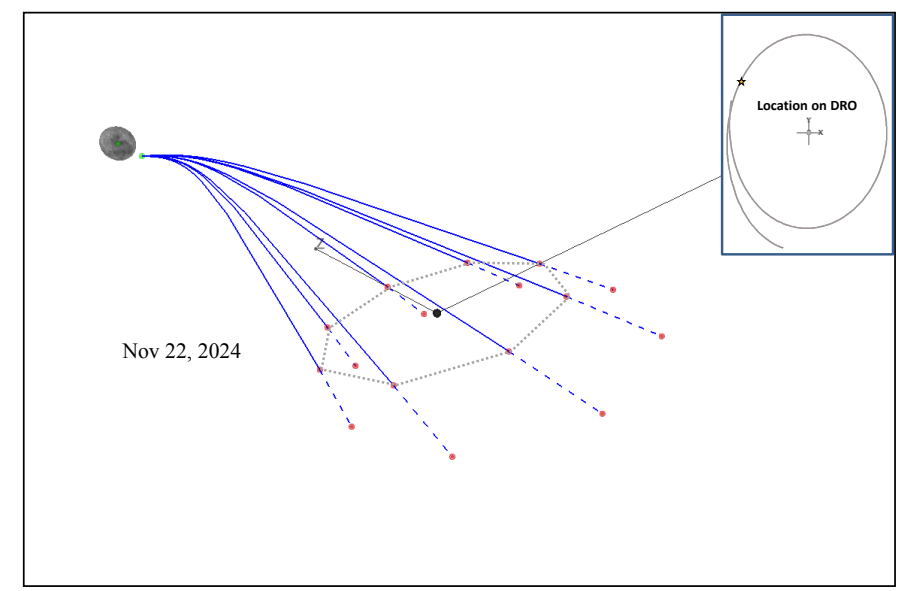

Figure 9b. November 22, 2024 maneuver from $20 \mathrm{~km}$ to $300 \mathrm{~m}$ offset 


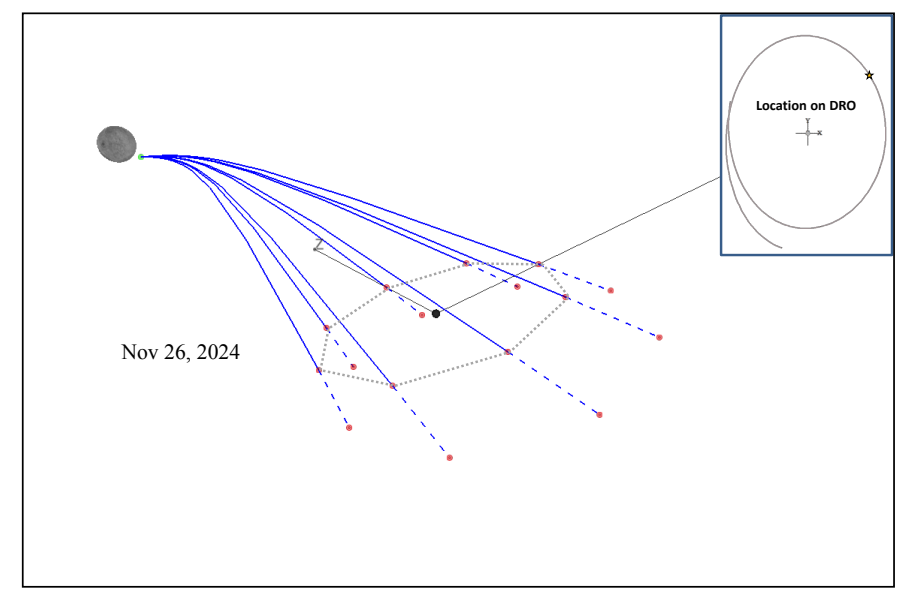

Figure 9c. November 26, 2024 maneuver from $20 \mathrm{~km}$ to $300 \mathrm{~m}$ offset

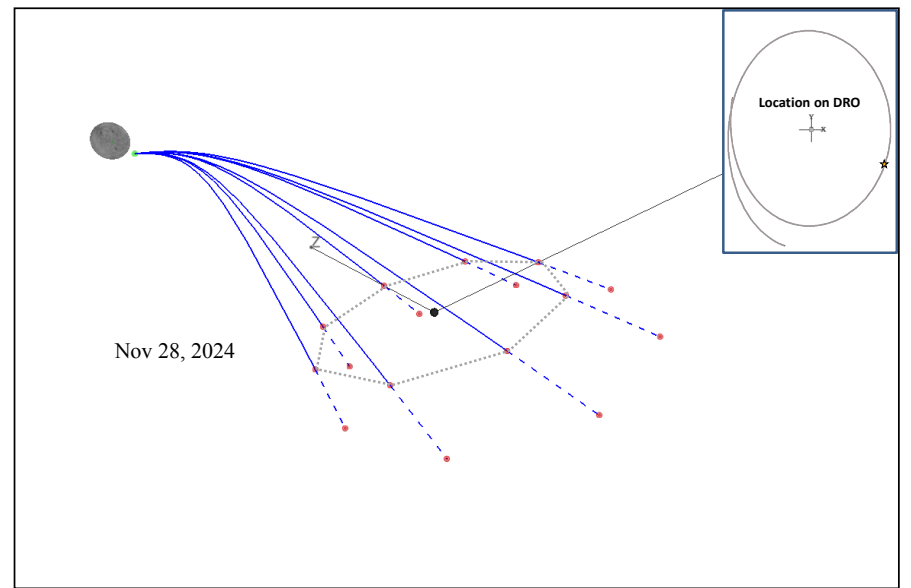

Figure 9d. November 28, 2024 maneuver from $20 \mathrm{~km}$ to $300 \mathrm{~m}$ offset

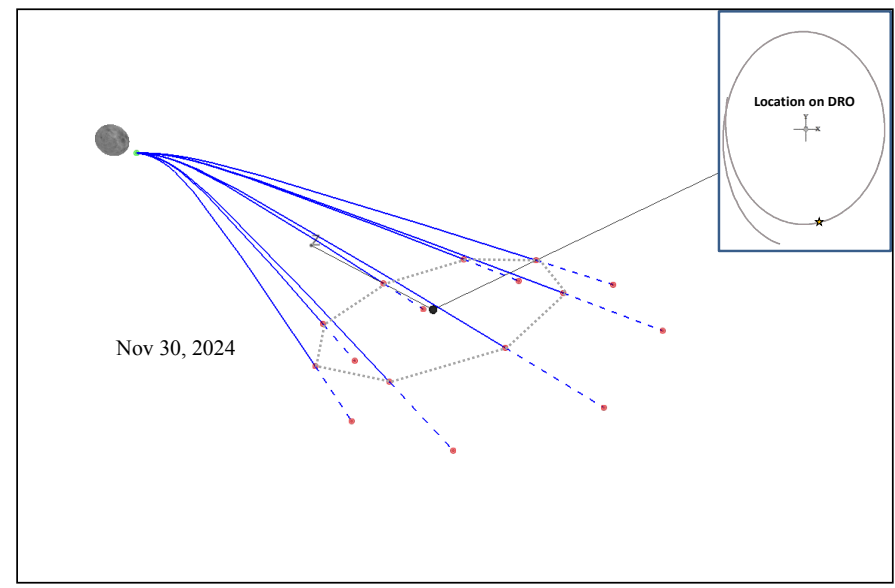

Figure 9e. November 30, 2024 maneuver from $20 \mathrm{~km}$ to $300 \mathrm{~m}$ offset

\section{Nominal Mission Rendezvous Strategy}

A nominal mid-field rendezvous mission transitions from the end of the far-field rendezvous at DRO insertion (see Figure 10). A $200 \mathrm{~m} / \mathrm{s}$ DRO capture maneuver places the Orion (Chaser) spacecraft $20 \mathrm{~km}$ below the ARV (Target) on the radial LVLH axis. After a 1 hour period for navigation state update and maneuver preparation, Orion 
performs a fundamentally two-burn sequence to close the distance between Chaser and Target from $20 \mathrm{~km}$ to a 300 $\mathrm{m}$ offset along a radial approach axis, which starts the prox-ops phase. A prox-ops phase takes Orion from the $300 \mathrm{~m}$ offset to docking, after which the crew spends approximately 5 days in mission operations with Orion docked to the ARV. The 5-day stay is followed by an Orion DRO departure burn targeted back to Earth via a lunar powered flyby.

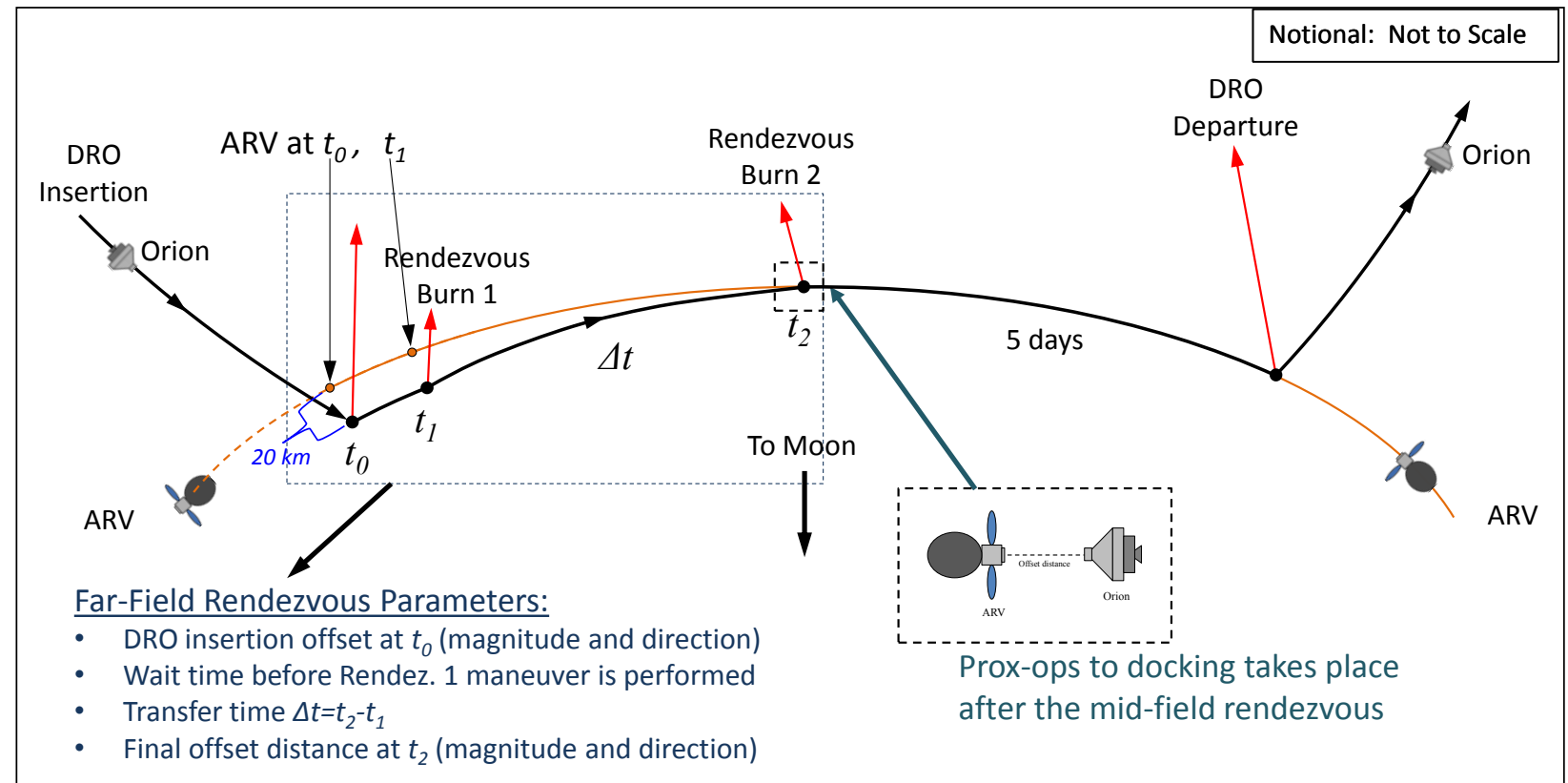

Figure 10. Nominal Mid-Field Rendezvous Mission

The near rectilinear motion during the mid-field rendezvous allows the location for the beginning of the prox-ops phase to be easily modified (see Figure 11). The trajectory can be modified to provide for Target collision avoidance in the event of a failed braking maneuver. The example in Figure X8 shows Orion arriving at the $300 \mathrm{~m}$ prox-ops Target offset distance with the sun in a preffered orientation (i.e., behind Orion as it approaches the Target). This approach also provides some measure of clearance from the Target to protect for a failed braking maneuver. This clearance can be enhanced with the additional maneuvers, thus creating a bent-pipe approach. This approach would be particularly helpful if, for example, the desired $300 \mathrm{~m}$ arrival distance from the Target were on the opposite side of the Target as Orion (at its initial $20 \mathrm{~km}$ distance). 


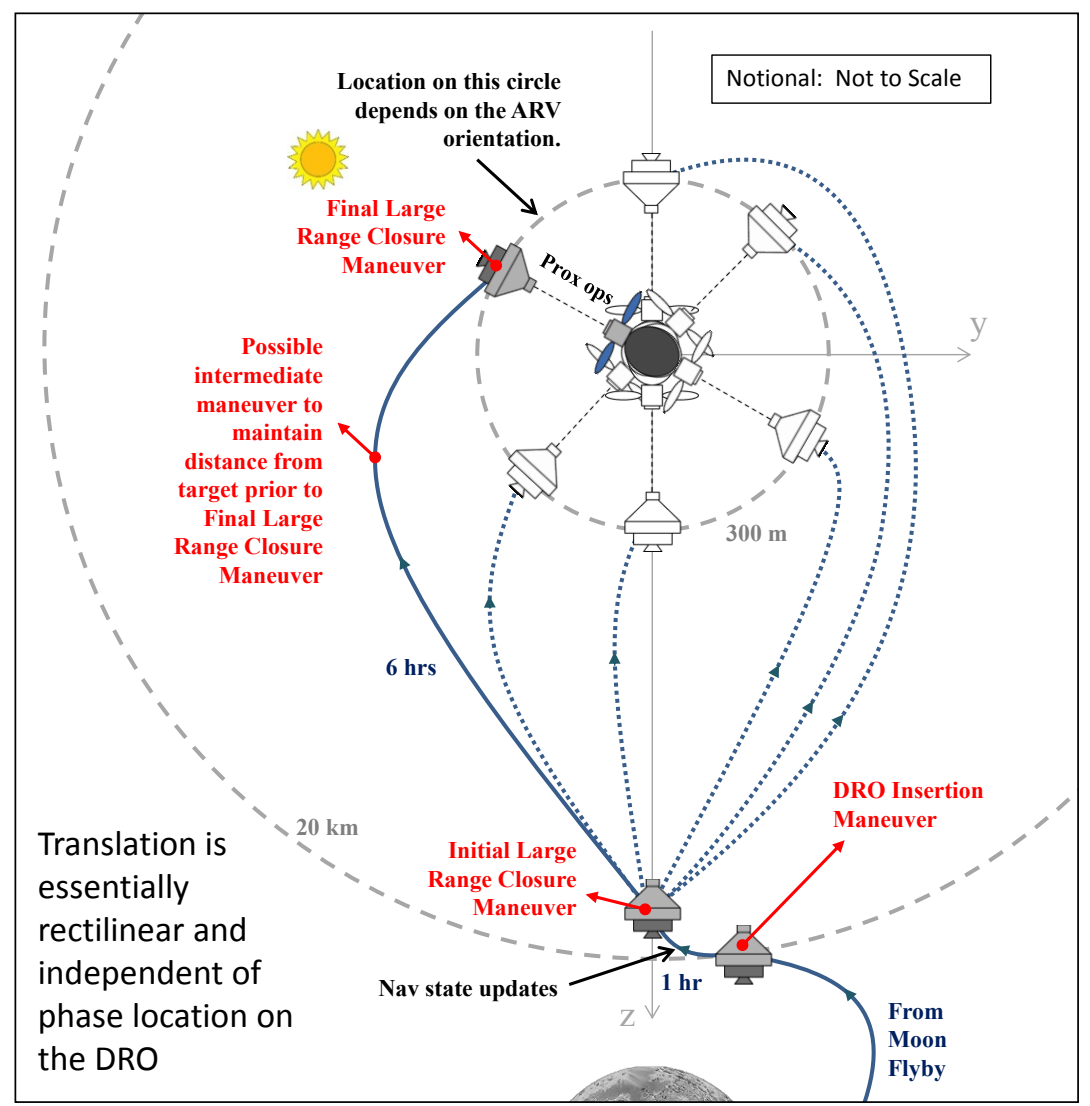

Figure 11. Nominal Mid-Field Rendezvous Mission. Includes example trajectory paths to variable $300 \mathrm{~m}$ distance positions.

\section{Sensor Conops}

The Orion RPOD with the ARV will require several navigation sensors and components, whether it is performed autonomously or with a pilot in the loop. This section describes the sensors and components required to successfully rendezvous and dock. Figure 18. shows the operational range for each sensor, along with a graphical depiction of their associated field of view (where applicable). The trajectory design and navigation filter, coupled with the sensor capabilities, will dictate the actual ranges that the sensors are enabled and utilized. Shown and described below is the approach as defined in Orion's RPOD baseline design.

Two star trackers and the S-band RF communications equipment are part of Orion's baseline to support nominal operations in Low-Earth Orbit (LEO) and beyond. The star trackers will be supporting long-range and mid-range bearing computation to a target vehicle, in addition to providing attitude updates to the Orion absolute navigation software. The S-band will provide range and range-rate measurements from the Orion to the ARV from 100km until the Laser Optical Camera Instrument (LOCI) is the primary RelNav sensor (approximately five to six kilometers). Depicted in the schematic within Figure 18., are the star trackers in the original configuration (Exploration Flight Test \#1, or EFT-1). The locations of the star trackers will change due to mass and ballast issues for future Orion Exploration Missions. 


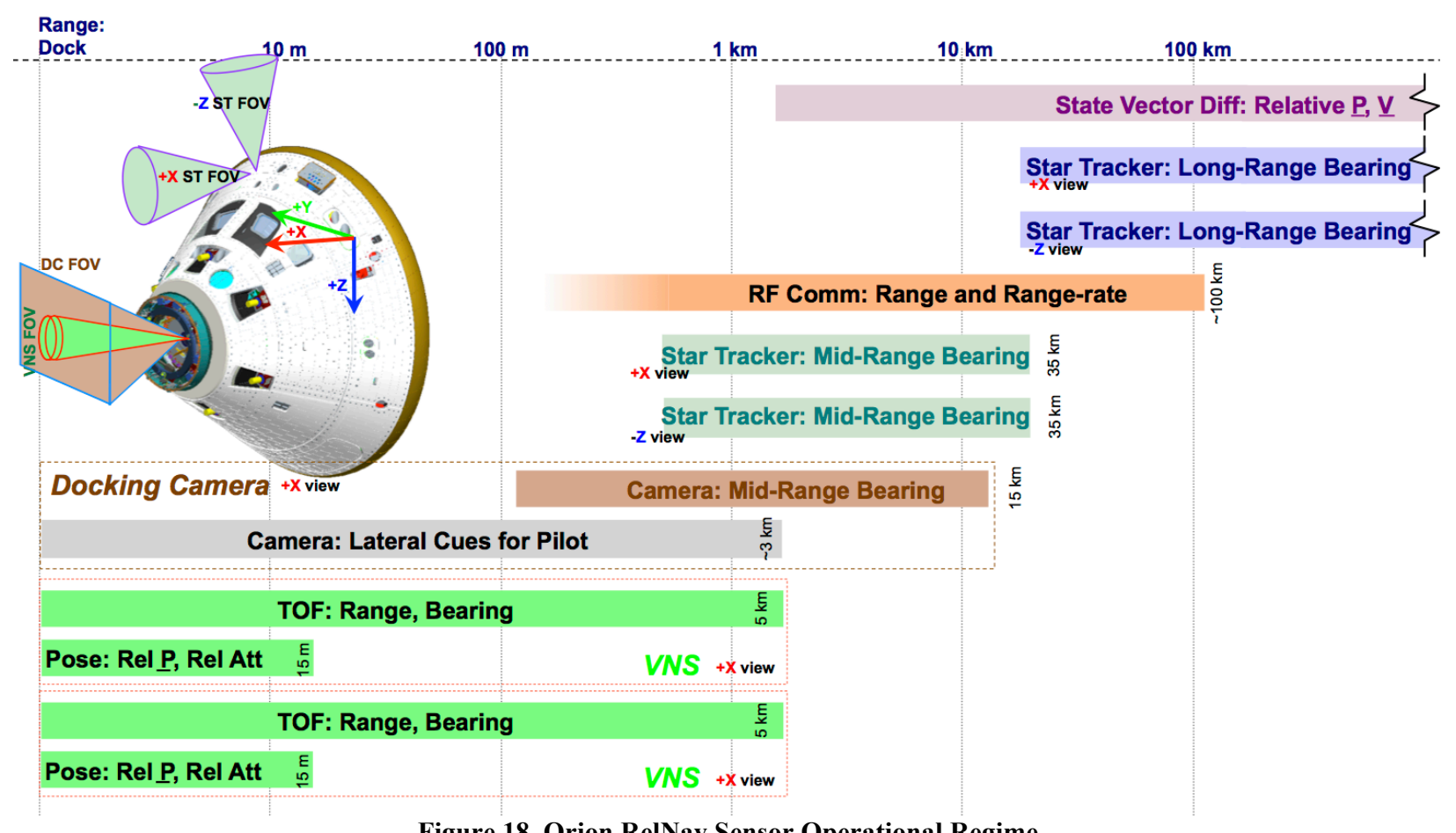

Orion's RelNav Kit, pictured in Figure 19., is comprised of two (2) Vision Navigation Sensors (VNS) and two (2) docking cameras into a single unit called the LOCI. The LOCI is a single chassis, dual one-fault tolerant sensor suite. LOCI is mounted to the docking hatch, operating through the docking hatch window (see Figure 19.Error! Reference source not found.). The crew installs and connects LOCI prior to performing any rendezvous and docking maneuvers at least one day prior to rendezvous operations. The LOCI will be activated around $5 \mathrm{~km}$ from the target vehicle, and should acquire the ARV shortly thereafter. The VNS component of the LOCI will measure the relative range and bearing from Orion to the ARV and provide the measurements to the RelNav filter. Simultaneously, the docking camera(s) within LOCI will provide additional imagery for the vision processing unit to perform centroiding on the image, generating a relative bearing measurement to supplement the VNS bearing measurements.

Once Orion is within 15 to 20 meters of the ARV, the VNS portion will be able to distinguish individual reflective elements (up to five "reflectors"). Based on the reflective element pattern, the Vision Processing Unit (VPU) computes a relative 6-DOF pose estimation (relative position and relative attitude) that is fed to the RelNav filter. Relative pose is a precise measurement of the target vehicle with respect to the chaser vehicle (ARV with respect to Orion) to enable the vehicles to perform fine alignment, prior to the final approach for docking. Simultaneously, the docking camera imagery is displayed for the crew to confirm the RelNav performance and that the vehicle is ready to proceed to docking.

Not shown in Figure 19. are the docking lights, which are located on the outside surface of the docking hatch, surrounding the docking hatch window. The docking lights illuminate the docking target on the ARV, to enable the docking camera component of the LOCI to operate independent of external lighting effects. Eveb though docking is expected to occur in sunlight, as the docking mechanisms get close, natural lighting of the docking target will be blocked. 


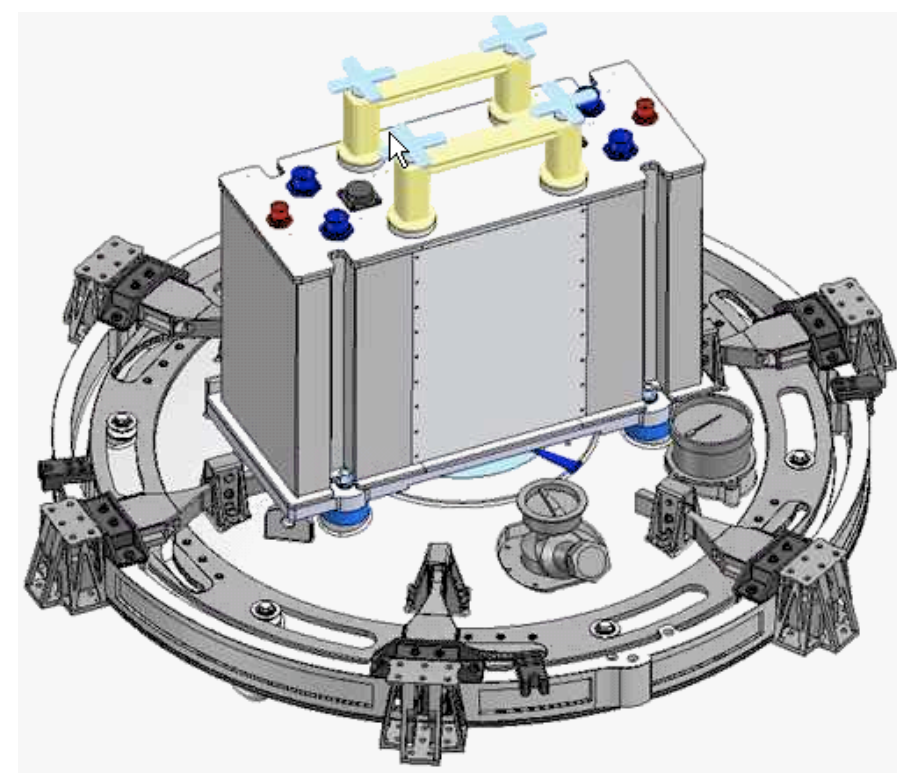

Figure 19. LOCI mounted to Orion docking hatch (interior view)

\section{Vision Navigation Sensor (VNS)}

The VNS portion of the LOCI is a laser-based, three-dimensional detector operating in the near infrared frequency band. The detector is comprised of an array of pixels that measure the laser light time of flight and the returned laser energy intensity. Coupling the detector with a specific set of lenses can ensure that the detector can have a field of regard covering $20^{\circ}$ (full angle). The VNS currently has an operational range of 5 kilometers to 1 meter, with a field-of-view of $\pm 6^{\circ}$ for long-range ( $5 \mathrm{~km}$ to $\left.1 \mathrm{~km}\right)$, and then enlarging to $\pm 10^{\circ}$ to support the midrange to close-range operations ( $1 \mathrm{~km}$ to $1 \mathrm{~m}$ ). Throughout the complete operational range, the VNS can output raw measurements at $30 \mathrm{~Hz}$. The raw measurements consist of the computed time of flight and the measured intensity for each pixel. Currently, the detector is an array of 256x256 pixels, whereby each pixel outputs a time of flight and an intensity measurement. In addition to the raw measurements, the VNS can output computed centroids to "bright" objects on the target vehicle. These outputs are computed at $1 \mathrm{~Hz}$ for long-range through short-range ( $5 \mathrm{~km}$ to $15 \mathrm{~m}$ ) and $5 \mathrm{~Hz}$ for short-range $(15 \mathrm{~m}$ to $1 \mathrm{~m})$. The long-range through short-range centroid output are measurements stated as relative range and relative bearing. The short-range measurements are stated as relative position and relative attitude, providing a relative six-degrees-of-freedom (6DOF) measurement. These measurements are passed into the RelNav filter for processing in support of performing RPOD.

\section{Docking Camera (DC)}

The primary purpose of the Docking Camera (DC) is to provide crew situational awareness of the target vehicle and to visually confirm that Orion is performing nominally during final approach. Current algorithm development and data processing from previous flight experiments are expanding the DC's utilization to be used as an OpNav device. Use of the DC as an OpNav camera relies on off-sensor image processing to generate a RelNav measurement. For long-range and mid-range to a target vehicle, the processed camera imagery generates a relative bearing (similar to the same measurement generated by a star tracker in tracking mode). For close-range applications, the processed DC imagery generates a RelNav solution. These measurements are typically generated with a known visual target or specific features on a target vehicle (sometimes referred to as Natural Feature Image Recognition or NFIR). To support these objectives, the DC has a wider field of view than the VNS, and will leverage off the latest advances in camera technology. Current specifications make use of a detector that has at least 4 mega-pixels, coupled with a fixed focal length optical lens $(8$ to $16 \mathrm{~mm}$ ) means that the DC can have a field of view in the $35^{\circ} \times 26^{\circ}$ to $19^{\circ} \times 15^{\circ}$ range (depending on lens selection). At full frame output, the current camera outputs at roughly $15 \mathrm{~Hz}$. Further analysis indicates a need for faster imagery output, the field of view must be reduced. Essentially, outputting a selective window region can be performed at much faster rates. The current limitation to frame size output rates are the interface buses used within the camera design. 


\section{Rendezvous Design}

\section{A. Navigation System Design}

Given the sensor suite outlined in Section XX, the navigation system will be designed to incorporate both passive and active sensors. The navigation system on Orion consists of two separate navigation filters: an Absolute Navigation Filter (ANF), which processes measurements from the Inertial Measurement Units (IMUs), the Star Trackers (STs) and navigation cameras, and a Relative Navigation Filter which, in addition to processing IMU data, processes bearing measurements from the $\mathrm{ST}$ and the navigation cameras, radiometric range and bearing from to the target vehicle, and LIDAR measurements which are range and bearing to reflectors. In addition, there will be a Relative Attitude Filter (RAF), which will operate from $15 \mathrm{~m}$ to dock.

The Orion RelNav Filter is an Extended Kalman Filter (EKF) which processes measurements from the Inertial Measurement Unit (IMU), ST/camera, radiometric range and range-rate, and the VNS. The Orion RelNav translation filter keeps two sets of inertial states: one set for Orion and another for the target vehicle. The states are: Orion position vector, Orion velocity vector, Orion attitude vector (consisting of Modified Rodrigues parameters), IMU bias and misalignment states, Star tracker misalignment, and the VNS biases. The Orion angular rate velocity is not a member of the state-space because attitude integration rates are provided by is done in the Orion IMU (OIMU). In the event that sensor data is lost, the filter can continue to propagate the two vehicles' states via dead reckoning so long as the OIMU data is available. The EKF processes $200 \mathrm{~Hz}$ data at $40 \mathrm{~Hz}$ intervals. Since there are multiple boxes associated with each sensor type, the sensor level Fault Detection, Isolation, and Recovery (FDIR) function selects the particular box to be used for that sensor type. The Star Tracker (ST) is the only purelybearing sensor used during Orion rendezvous operations. In practice the RelNav EKF will initialize the Orion state from the selected) the Absolute Navigation filter slaved to a particular IMU. The target state will be initialized based upon an uploaded target ephemeris, with the assumption that the target is not a maneuvering vehicle.

The translation RelNav filter differs from a generic EKF in two important ways. First, the Orion attitude states are handled in the manner of a Multiplicative EKF (MEKF). The second is that measurements that are received by the filter at the same time (though the time-tags of the measurements can vary due to measurement and timing latencies) are processed in the manner of a linear Kalman filter (KF), with a state update in the manner of an EKF occurring only after all of the aforementioned measurements are processed. The linear update was adopted due to the well-known difficulty that arises in the use of an EKF when combining some very precise measurements with measurements that are less precise (like inertial measurements). The nonlinearity of the relative measurements results in different filter solutions for a different ordering of measurement processing, and can even cause the EKF to diverge. This order dependency is mitigated by using a hybrid linear/extended Kalman Filter, whereby groups of measurements (inertial and relative) which are received at the same time, are processed as in a linear KF, after which the propagated state is updated in the familiar EKF sense.

The filter utilizes a second-order under-weighting process to account for the nonlinearities inherent in accurate range and bearing measurements. A Sigma-Point Kalman Filter (SPKF) was considered but was not further pursued due to the fact that it yields the same benefit as the second-order filter, while requiring more computations.

Finally, there is a RAF that operates when the two vehicles are closer than 15 meters apart. This filter is a 9-state filter containing the relative attitude of the target, the attitude rate of the target and three misalignments. The sensor measurement is the attitude component of the pose measurement. Unlike the RelNav translation filter, this filter is very simple having to only process the pose obtained from the VNS.

\section{Rendezvous Testing}

\section{A. STORRM}

A prototype system, called Sensor Test for Orion RelNav Risk Mitigation (STORRM), consisting of a VNS and a docking camera (DC), was tested on STS-134. The prototype system was demonstrated during docking, undocking and re-rendezvous operations. The STORRM package was installed on the Orbiteter Docking System (ODS) adjacent to the Trajectory Control Sensor (TCS). The VNS and DC were operated at various ranges and phases of the Orbiter's trajectory on flight days 3 (rendezvous and docking) and 15 (undocking, re-rendezvous and departure). NEED TO STATE THAT THERE ARE MULTIPLE PAPERS ON THIS SUBJECT! 


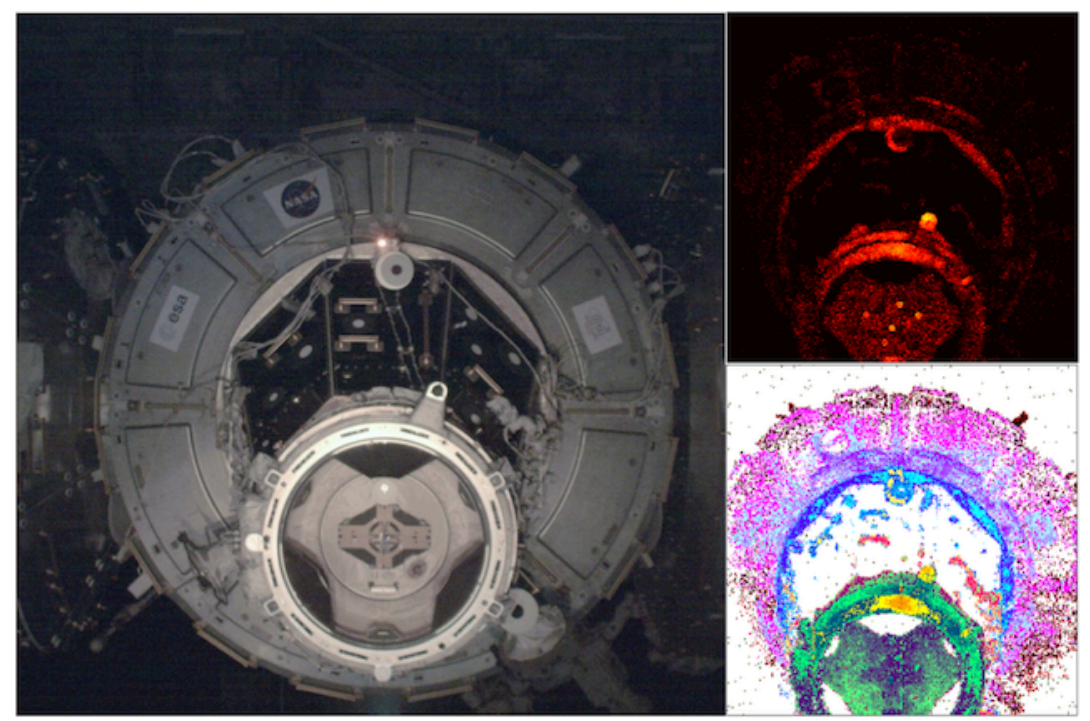

Figure 20.STORRM Imagery -- Docking camera to left, VNS intensity top right, VNS range bottom right

\section{B. SOSC Testing}

Several test campaigns have been conducted over the past several years in the Lockheed Martin Space Operations and Simulation Center (SOSC) facility. The initial objectives for Orion testing in the SOSC were twofold: first to characterize the facility resources (motion control, target environment, lighting, etc.) as providing a space-like environment for future Orion testing, and second to develop a core capability to execute a closed-loop Orion rendezvous and docking simulation in the SOSC. The first objective is discussed extensively by Christian et. al. [1].

The main objective of these latter tests were to demonstrate the effectiveness of the VNS operating in a closedloop with other elements of the RPOD system, including the navigation filter, the guidance system, and the control system. These tests were limited to the range of $55 \mathrm{~m}$ to dock with ISS (subsequent tests will evaluate the system from $60 \mathrm{~m}$ to dock). In addition, off-axis tests were conducted; these tests evaluated the ability of the system to handle trajectory dispersions.

The Orion program conducted testing in the SOSC in July 2013 in order to execute a closed-loop rendezvous simulation using Guidance, Navigation, and Control (GN\&C) flight software (FSW) algorithms developed by the Orbit MODE Team (OMT). The test included executing the GN\&C flight software algorithms closed-loop with the VNS unit that was flown on the Space Shuttle (STS-134) Development Test Objective (DTO) earlier in the year in STORRM. While the DTO tested performance of the Orion RelNav hardware in a space environment, it did not test the overall performance of the Orion RelNav system. The purpose of testing in the SOSC facility was to take the DTO one step further and characterize the performance of the entire Orion RelNav system in various Orion docking profiles.

In January 2014, the Orion program conducted another series of tests in the SOSC to (1) characterize long range performance of the VNS and (2) characterize performance of the VNS behind multiple panes of glass. The results of these tests are still being analyzed but the information gathered will be valuable moving forward with the Orion relative navigation sensor design.

These tests were only the beginning of what will be required to develop rendezvous capability for the Orion vehicle, but it gave the team the opportunity to integrate the software and hardware components of the RelNav system for the first time.

The testing to date has demonstrated that the proximity operations and docking phase of the mission, which has been tested in the SOSC, is rapidly maturing. Extensive tests have increased confidence in the functionality, performance and robustness of the design.

\section{Integrated Power, Avionics, and Software (iPAS)}

The Integrated Power, Avionics, and Software (iPAS) environment at the Johnson Space Center (JSC) was developed to support technology maturation and capabilities development for human spaceflight. To evaluate these 
capabilities for future use, tests are conducted periodically within iPAS that demonstrate aspects of proposed future missions. In 2013, the iPAS team executed a demonstration of Orion docking with the ARV in a DRO.

In order to conduct a closed-loop AR\&D demonstration, a simulation of the 6 Degree of Freedom (6DOF) dynamics environment at DRO was required. The iPAS team leveraged an existing ARM simulation within the Trick Simulation Environment, developed by a team lead by Edwin Z. (Zack) Crues. This Trick simulation was used to perform early analysis and trades for JSC engineers working ARM concept development. The simulation included a simple model of the Jet Propulsion Laboratory (JPL) vehicle, which for this test only performed attitude control sufficient to support the AR\&D operations. The Orion vehicle was also modeled, including the use of the ATVderived Service Module. Simple range, range-rate, and bearing sensors were used to provide inputs to flight software.

The manual control system supplied with the Trick simulation was replaced with an early version of the Orion AR\&D software developed by the OMT and by Draper Labs. Parts of the AR\&D algorithms were expressed within the Simulink analysis tool, where closed loop design evaluation could be performed. The FSW was autocoded from these Simulink diagrams, in a process similar to how the actual Orion FSW is being developed by NASA and Lockheed. The AR\&D guidance was modified to account for the dynamic environment expected at DRO, where the affects of a central body are much smaller compared to LEO. This modification work was performed by Juan Senent/Odyssey. The navigation and autonomous control elements were then integrated into a GN\&C application delivery by Matt Fritz/Draper Labs.

A software framework called Core Flight Software (CFS) was selected to run the AR\&D software, since there was not sufficient expertise to use the Rhapsody tool selected by Lockheed for Orion. So while the integration code was not Orion like, the GN\&C applications themselves were faithful to the Orion GN\&C process. CFS was developed by Goddard Space Flight Center (GSFC) and the delivery to iPAS was performed by Lore Prokop/JSC and Steve Duran/JSC. The flight software product was integrated by Teming Tse/JSC. For this first test, both the simulation and the CFS flight software were executed on a Linux computer (CentOS 6.5). Soon this CFS executable will be targeted for a variety of PPC-750-based flight-like processors within iPAS.

The simulation was augmented to support real-time performance analysis, by Andrew Spencer/Odyssey. Using a Trick service called IO Channels, the data interfaces between simulated vehicle systems and the flight software were established. Through these channels, the relative sensor data, calculated based on the 6DOF dynamics environment in Trick, was sent to the AR\&D algorithms running in CFS. Then the jet commands from the AR\&D GNC were sent back to the Trick simulation, for modeling of jet firing and integration with the dynamic environment.

A proposed mission scenario was developed: not a baseline for the mission, but a credible AR\&D profile that would demonstrate feasibility. The Orion vehicle was position $2 \mathrm{~km}$ away from the ARV/asteroid stack that was prepositioned in DRO and was performing attitude control. The AR\&D FSW commanded a rendezvous burn to a location $300 \mathrm{~m}$ "in front of" the JPL vehicle along the docking system centerline axis, where a planned stationkeeping was performed. The next phase of approach was initiated, and the Orion continued along the centerline axis to a location $10 \mathrm{~m}$ from the ARV docking adaptor, where another stationkeep was commanded. Finally the Orion was commanded to conduct final approach and docking. For this test, the docking was complete based only on range; plans to integrate early models of the NASA Docking System (NDS) are being discussed.

It should be noted that this test did not baseline any particular mission elements. The value of this demonstration was the application of Orion-like flight software to this new mission scenario. Valuable insights were gained developing the closed-loop simulation, modifying the flight software to account for the mission environment, and demonstrating the execution of autonomous aspects. Future improvements to this testing would include more complete models of the proposed AR\&D relative sensor suite, as well as integration of Orion Exploration Mission 1 (EM1) autocode. Work is underway to provide closed-loop integrated testing with physical cameras and star trackers, that are stimulated by star field models sycnronized with the environment in the 6DOF simulation. The output of these sensor models will be fed directly into the Orion-like FSW.

\section{ISS STP-H5 Raven}

NASA-GSFC's Satellite Servicing Capabilities Office (SSCO) has proposed an experiment to be flown to ISS on the US Air Force's Space Test Program - Houston 5 (STP-H5) mission. This experiment, called Raven, will consist of flash LIDAR, infrared camera and optical camera. All three sensors will be mounted in a single housing that will be affixed to a gimbaled platform on an ISS Express Logistics Carrier (ELC) which will be installed on the ISS truss segment. The objective of the Raven experiment is to advance the rendezvous and proximity operations hardware and software in a space environment. Raven will utilize the same VNS that was flown on STS-134 as part of the STORRM DTO, and has been used frequently in the SOSC testing. There will be some minor adjustments made to 
the VNS prior to launching in early 2016. By utilizing the VNS, IR and visible cameras, the Raven experiment will further enhance NASA's capabilities in both cooperative and non-cooperative relative navigation; which will be applied to ARCM.

\section{Rendezvous Design Forward Work}

NASA needs AR\&D sensors for both the robotic and crewed segments of the ARM. NASA recently conducted a commonality assessment of the concept of operations for the both the robotic ARV concepts and the crewed mission segment concept using the Orion crew vehicle. The commonality assessment also considered several future exploration and science missions requiring an AR\&D capability such as asteroid sample return, satellite servicing, and planetary entry, descent, and landing. This assessment determined that a common sensor suite consisting of one or more visible wavelength cameras, a three-dimensional LIDAR along with a long-wavelength infrared camera for robustness and situational awareness could be used on each mission to eliminate the cost of multiple sensor developments and qualifications. By choosing sensor parameters at build time instead of at design time and, without having to requalify flight hardware, a specific mission can design overlapping bearing, range, relative attitude, and position measurement availability to suit their mission requirements with minimal nonrecurring engineering costs. The resulting common sensor specification provides the union of all performance requirements for each mission and represents an improvement over the current systems used for AR\&D today. Table B summarizes the concept of operations for each of the asteroid mission concepts and how that mission will apply sensors from the common suite.

Table B. AR\&D Concepts of Operations for Asteroid Mission Concepts

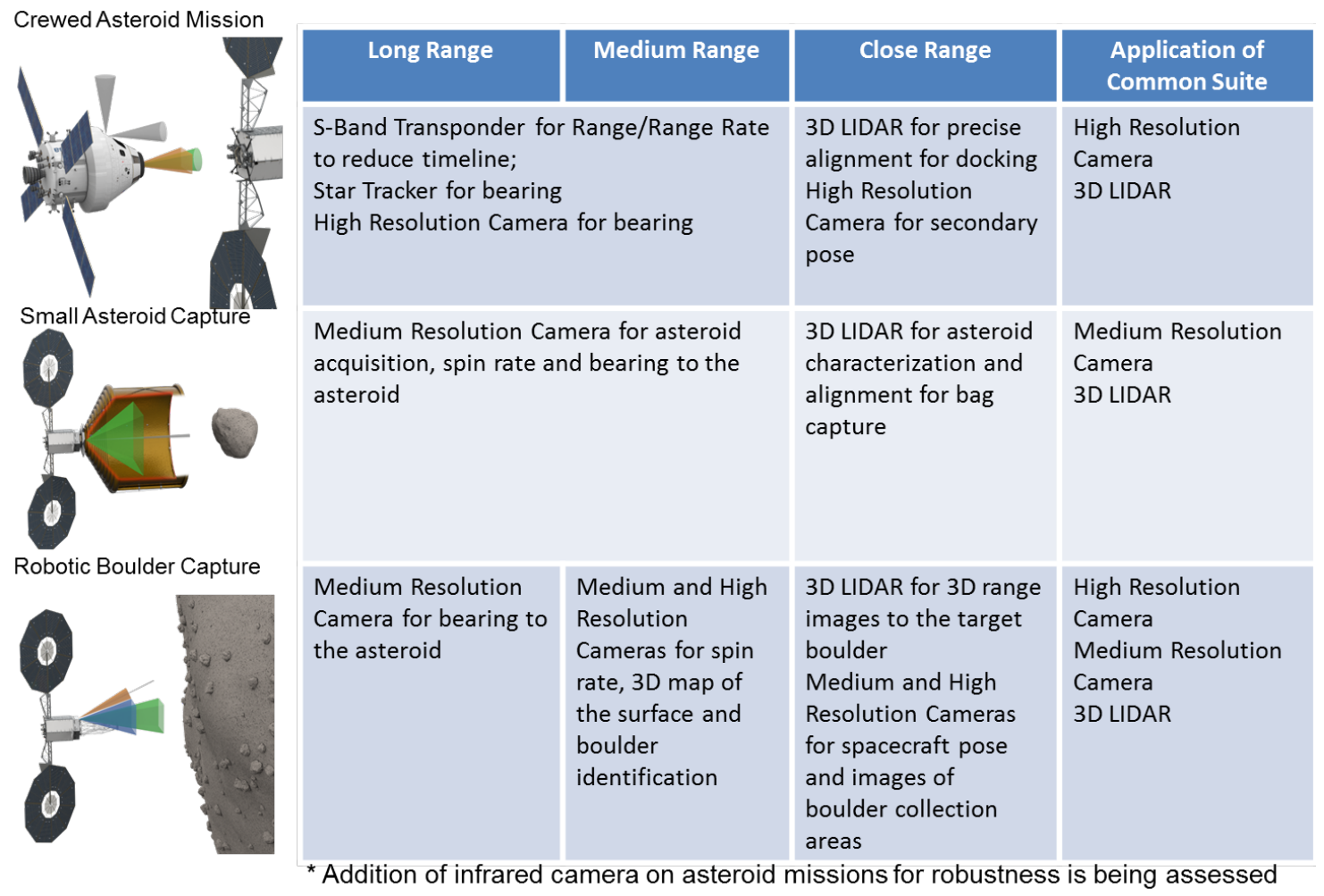

The common specification identified a set of environmental requirements common to each concept of operation. These values are shown below in Table B-1. Where possible, it was noted where an attribute could be instantiated in a modular fashion, or where the sensor design includes build-time options that can change its intended use. One example is target albedo: a sensor built for the ARV could be assembled differently than one assembled for Orion in order to meet the low albedo of the asteroid as opposed to the potentially highly reflective target on the ARV. While 
such modularity works for the asteroid applications covered here, extension to other future missions such as satellite servicing may require both ends of the attribute spectrum.

Table B-1: Environmental Commonality Specification

\begin{tabular}{|c|c|c|c|}
\hline Attribute & Units & Specification & Notes \\
\hline Operational Regime & NA & $\begin{array}{l}\text { Deep space and } \\
\text { cis-lunar }\end{array}$ & \\
\hline Mission Duration & Years & $>7$ & \\
\hline Sensor On-time & Hours & $\begin{array}{l}>1600 \text { (not for all } \\
\text { sensors in the } \\
\text { suite) }\end{array}$ & $\begin{array}{l}\text { The suite is used as described in the } \\
\text { supporting materials on the BAA website. } \\
\text { Accounting for duty cycling, the on-time } \\
\text { could be shorter. }\end{array}$ \\
\hline $\begin{array}{l}\text { Operational Thermal } \\
\text { Range }\end{array}$ & $\operatorname{deg} \mathrm{C}$ & -30 to +50 & $\begin{array}{l}\text { Survival temperature range should be wider } \\
\text { than the operational. }\end{array}$ \\
\hline Tested Partial Pressure & $\mathrm{Pa}$ & $<1 \mathrm{e}-5$ & $\begin{array}{l}\text { The actual environment will be a hard } \\
\text { vacuum. }\end{array}$ \\
\hline Total Ionizing Dose $\left(^{*}\right)$ & kRad Si & $>100$ & $\begin{array}{l}\text { Computed TID should account for sensor } \\
\text { on-time and mission elapsed time, which } \\
\text { are different from each other }\end{array}$ \\
\hline Single Event Upset Rate & Upsets/day & $<1 \mathrm{e}-2$ & $\begin{array}{l}\text { Computed rates should be for functional } \\
\text { upsets only that require a power cycle or } \\
\text { configuration reload from stored memory. }\end{array}$ \\
\hline Asteroid Size & Meters & $2-500$ & $\begin{array}{l}\text { Small sizes apply to reference mission and } \\
\text { larger sizes apply to alternate }\end{array}$ \\
\hline $\begin{array}{l}\text { Asteroid visible albedo } \\
(*)\end{array}$ & $\%$ & $>3$ & $\begin{array}{l}\text { Depends on material make up with } 3 \% \\
\text { being a minimum }\end{array}$ \\
\hline $\begin{array}{l}\text { Docking target } \\
\text { reflectance }(*)\end{array}$ & $\%$ & $>90$ & Docking target to use retro-reflectors \\
\hline Sun Exposure Survival & Hours & Indefinite & No requirement to operate with Sun in view \\
\hline
\end{tabular}

The top-level performance attributes required to meet all three of the mission concept of operations, for each type of sensor, is given in Table B-2.

Table B-2: Commonality Specification for Each Sensor Type

\begin{tabular}{|l|l|l|l|l|}
\hline & \multicolumn{1}{|c|}{$\begin{array}{c}\text { Visible } \\
\text { Camera }\end{array}$} & \multicolumn{1}{c|}{$\begin{array}{c}\text { Infrared } \\
\text { Camera }\end{array}$} & \multicolumn{1}{c|}{ LIDAR } & \multicolumn{1}{c|}{ Notes } \\
\hline $\begin{array}{l}\text { Minimum } \\
\text { Operational }\end{array}$ & $1 \mathrm{~m}$ & $1-2 \mathrm{~m}$ & $1 \mathrm{~m}$ & $\begin{array}{l}\text { Ranges closer than } 1 \mathrm{~m} \\
\text { are being explored for } \\
\text { Orion may be addressed } \\
\text { by proposers. }\end{array}$ \\
\hline $\begin{array}{l}\text { Maximum } \\
\begin{array}{l}\text { Operational } \\
\text { Range }\end{array}\end{array}$ & $\begin{array}{l}>50,000 \mathrm{~km} \\
\text { (bearing only) }\end{array}$ & $\begin{array}{l}100 \mathrm{~m} \text { to } 200 \mathrm{~km} \\
\text { (bearing only) }\end{array}$ & $\begin{array}{l}2-3 \mathrm{~km} \text { (range and } \\
\text { bearing) }\end{array}$ & $\begin{array}{l}\text { LIDAR: } \\
2-3 \mathrm{~km} \text { initial detection } \\
\text { range. }\end{array}$ \\
\hline
\end{tabular}




\begin{tabular}{|c|c|c|c|c|}
\hline $\begin{array}{l}\text { Operational Field } \\
\text { of View }\end{array}$ & $\begin{array}{l}\text { Rendezvous: } \\
0.5-1.5 \text { deg } \\
\text { Prox Ops: } \\
30-45 \text { deg }\end{array}$ & $\begin{array}{l}\text { Typically max of } \\
30 \text { and min of } 20^{\circ} \text {, } \\
\text { but is application } \\
\text { dependent. Ability } \\
\text { to utilize } \\
\text { appropriate lenses } \\
\text { required. }\end{array}$ & $\begin{array}{l} \pm 30^{\circ} \text { ARV } \\
\pm 10^{\circ} \text { Orion }\end{array}$ & $\begin{array}{l}\text { LIDAR: } \\
\text { ARV requires } \pm 30^{\circ} \text { to } \\
\text { "capture" target, ensuring } \\
\text { bag does not snag. } \\
\text { Orion requires } \pm 10^{\circ} \text { to } \\
\text { ensure relative } \\
\text { measurement accuracy } \\
\text { throughout } \mathrm{AR} \& D / C \text {. }\end{array}$ \\
\hline $\begin{array}{l}\text { Angular } \\
\text { Resolution }\end{array}$ & $\begin{array}{l}\text { Rendezvous: } \\
<8 \text { urad/pixel } \\
\text { Prox Ops: } \\
<300 \text { urad/pixel }\end{array}$ & $\begin{array}{l}\text { Prox Ops: } \\
<300 \mathrm{urad} / \text { pixel }\end{array}$ & $\begin{array}{l}<1.5 \text { mrad } \\
\text { /measurement }\end{array}$ & \\
\hline Range Accuracy & NA & NA & $\begin{array}{l}\text { Precision: } 2 \mathrm{~cm}(1- \\
\text { sigma) within a } \\
\text { frame } \\
\text { Accuracy: } 2 \mathrm{~cm}(1- \\
\text { sigma) at } 2 \mathrm{~m} \\
\text { separation }\end{array}$ & \\
\hline Wavelengths $(*)$ & $400-700 \mathrm{~nm}$ & $8-14$ um & $\begin{array}{l}\text { ARV: no restrictions } \\
\text { Orion: Eye safe }\end{array}$ & $\begin{array}{l}\text { Eye safety could be met } \\
\text { with system protections or } \\
\text { laser output frequency }\end{array}$ \\
\hline Frame Rates & $5-10 \mathrm{~Hz}$ & $3-5 \mathrm{~Hz}$ & $5-10 \mathrm{~Hz}$ & \\
\hline Dynamic Range & $>1000: 1$ & $>1000: 1$ & NA & For a single exposure \\
\hline
\end{tabular}

NASA acknowledges that as technology improves, changes, and matures, the common sensor suite will need to change and adapt. NASA invites innovative ideas for how to incrementally improve this sensor suite as technology improves and advances. Examples include advances in the regime of noise radar, computing, and other technologies that could enable new approaches to AR\&D. New technologies could result in savings on size, weight, power, and complexity. NASA would like to establish incremental growth in AR\&D/C capability including the ability to operate in parallel to other solutions to demonstrate the technology prior to integrating it into the primary solution set.

Alternate ARV attitude strategy during RPOD.

Further analysis is needed to fully address control of bright objects for the optical sensors.

Dispersion analysis to size offsets, place mid-course maneuvers, and assess integrated system performance.

\section{Appendix [Do we need this??]}

An appendix, if needed, should appear before the acknowledgements.

\section{Acknowledgments [Volunteer author?]}

The preferred spelling of the word "acknowledgment" in American English is without the "e" after the "g." Avoid expressions such as "One of us (S.B.A.) would like to thank..." Instead, write "F. A. Author thanks..." Sponsor and financial support acknowledgments are also to be listed in the "acknowledgments" section.

Zack Crues/JSC, Paul Bielski/JSC, Hung Nguyen/L-3 Corp, Matt Fritz/Draper, Juan Senent/Odyssey, Lore Prokop/JSC, Steve Duran/JSC, Teming Tse/JSC 